\title{
Irrationalité aux entiers impairs positifs d'un $q$-analogue de la fonction zêta de Riemann
}

\author{
Frédéric Jouhet et Elie Mosaki
}

2000 Mathematics Subject Classification: Primary 11J72; Secondary 33D15.

Key words and phrases: q-analogue de la fonction zêta de Riemann, irrationalité, séries hypergéométriques basiques.

Résumé. Dans cet article, nous nous intéressons à un $q$-analogue aux entiers positifs de la fonction zêta de Riemann, que l'on peut écrire pour $s \in \mathbb{N}^{*}$ sous la forme $\zeta_{q}(s)=\sum_{k \geq 1} q^{k} \sum_{d \mid k} d^{s-1}$. Nous donnons une nouvelle minoration de la dimension de l'espace vectoriel sur $\mathbb{Q}$ engendré, pour $1 / q \in \mathbb{Z} \backslash\{-1 ; 1\}$ et $A$ entier pair, par $1, \zeta_{q}(3), \zeta_{q}(5), \ldots, \zeta_{q}(A-1)$. Ceci améliore un résultat récent de Krattenthaler, Rivoal et Zudilin (Séries hypergéométriques basiques, q-analogues des valeurs de la fonction zêta et séries d'Eisenstein, J. Inst. Jussieu 5.1 (2006), 53-79). En particulier notre résultat a pour conséquence le fait que pour $1 / q \in \mathbb{Z} \backslash\{-1 ; 1\}$, au moins l'un des nombres $\zeta_{q}(3), \zeta_{q}(5), \zeta_{q}(7), \zeta_{q}(9)$ est irrationnel.

Abstract. In this paper, we focus on a $q$-analogue of the Riemann zêta function at positive integers, which can be written for $s \in \mathbb{N}^{*}$ by $\zeta_{q}(s)=\sum_{k \geq 1} q^{k} \sum_{d \mid k} d^{s-1}$. We give a new lower bound for the dimension of the vector space over $\mathbb{Q}$ spanned, for $1 / q \in \mathbb{Z} \backslash\{-1 ; 1\}$ and an even integer $A$, by $1, \zeta_{q}(3), \zeta_{q}(5), \ldots, \zeta_{q}(A-1)$. This improves a recent result of Krattenthaler, Rivoal and Zudilin (Séries hypergéométriques basiques, q-analogues des valeurs de la fonction zêta et séries d'Eisenstein, J. Inst. Jussieu 5.1 (2006), 53-79). In particular, a consequence of our result is that for $1 / q \in \mathbb{Z} \backslash\{-1 ; 1\}$, at least one of the numbers $\zeta_{q}(3), \zeta_{q}(5), \zeta_{q}(7), \zeta_{q}(9)$ is irrational.

\section{Introduction}

L'étude de l'irrationalité des valeurs de la fonction zêta de Riemann $\zeta$ aux entiers impairs positifs est un problème classique en théorie des nombres. Il 
est en effet connu que l'expression des valeurs de $\zeta$ aux entiers pairs positifs

$$
\zeta(2 m)=(-1)^{m-1} 2^{2 m-1} B_{2 m} \frac{\pi^{2 m}}{(2 m) !}
$$

permet d'affirmer, via la transcendance de $\pi$, due à Lindemann, que chacun de ces nombres est transcendant (ici $m \in \mathbb{N}^{*}$ et les nombres rationnels $B_{m}$ sont les nombres de Bernoulli). En revanche, concernant l'étude aux entiers impairs positifs, même si la transcendance est conjecturée, le seul résultat significatif fût pendant longtemps le théorème d'Apéry [3] affirmant que $\zeta(3)$ est irrationnel. Puis récemment, Rivoal [16], et Ball et Rivoal [6] ont eu l'idée de considérer les valeurs de $\zeta$ aux entiers impairs positifs dans leur ensemble plutôt qu'individuellement, ce qui leur permit de prouver qu' il existe parmi les nombres $\zeta(2 m+1), m \in \mathbb{N}^{*}$, une infinité de nombres irrationnels, en donnant la minoration pour $A$ entier pair suffisamment grand :

$$
\operatorname{dim}_{\mathbb{Q}}(\mathbb{Q}+\mathbb{Q} \zeta(3)+\cdots+\mathbb{Q} \zeta(A-1)) \geq \frac{\log A}{1+\log 2}(1+\mathrm{o}(1)) .
$$

La méthode employée a conduit à des versions quantitatives [6, 11, 16, 18, jusqu'à l'article récent de Zudilin [22] dans lequel il est prouvé qu' au moins l'un des nombres $\zeta(5), \zeta(7), \zeta(9), \zeta(11)$ est irrationnel. Le lecteur intéressé pourra aussi consulter le survol de Fischler [8] sur ce sujet.

Dans cet article, nous nous intéressons au $q$-analogue normalisé de la fonction $\zeta$ considéré d'abord dans [10] et [23], puis plus récemment encore dans [12, et que l'on peut écrire pour $s \in \mathbb{N}^{*}$ et $q$ un nombre complexe tel que $|q|<1$ :

$$
\zeta_{q}(s)=\sum_{k \geq 1} q^{k} \sum_{d \mid k} d^{s-1}=\sum_{k \geq 1} k^{s-1} \frac{q^{k}}{1-q^{k}} .
$$

Le terme de $q$-analogue est justifié ici par la relation valide pour $s \in \mathbb{N}^{*} \backslash\{1\}$ (voir par exemple [10] ou [12] pour une démonstration) :

$$
\lim _{q \rightarrow 1}(1-q)^{s} \zeta_{q}(s)=(s-1) ! \zeta(s),
$$

où bien entendu $\zeta(s)=\sum_{k>1} \frac{1}{k^{s}}$ est l'expression pour $R e(s)>1$ de la fonction zêta de Riemann. L'un des intérêts de ce $q$-analogue de $\zeta$ réside dans le fait que les valeurs de $\zeta_{q}$ aux entiers pairs positifs sont reliées aux formes modulaires et aux séries d'Eisenstein $E_{2 m}(q)\left(m \in \mathbb{N}^{*}\right)$ [19] via la relation :

$$
E_{2 m}(q)=1-\frac{4 m}{B_{2 m}} \zeta_{q}(2 m)
$$


Concernant la transcendance des valeurs de $\zeta_{q}$ aux entiers pairs positifs, le résultat définitif est conséquence de la structure de l'espace des formes modulaires sur $S L_{2}(\mathbb{Z})[19$ ] et d'un théorème d'indépendance algébrique sur les séries d'Eisenstein $E_{2}(q), E_{4}(q)$ et $E_{6}(q)$ dû à Nesterenko [14]. En effet, on peut déduire de cela que pour $m \in \mathbb{N}^{*}$ et $q$ algébrique (en particulier $1 / q \in \mathbb{Z} \backslash\{-1 ; 1\})$, les nombres $\zeta_{q}(2 m)$ sont tous transcendants.

Ceci conduit naturellement à se pencher sur le cas des valeurs de $\zeta_{q}$ aux entiers impairs positifs. Remarquons tout d'abord que malgré l'analogie manifeste entre les résultats de transcendance des valeurs de $\zeta$ et $\zeta_{q}(1 / q \in$ $\mathbb{Z} \backslash\{-1 ; 1\})$ aux entiers pairs positifs, il n'est aujourd'hui possible d'affirmer l'irrationalité de $\zeta_{q}(3)$ pour aucune valeur de $q$. En fait, seule l'irrationalité de $\zeta_{q}(1)$ est connue [5] pour diverses valeurs de $q$. D'autre part, on sait depuis [15] que $1, \zeta_{q}(1), \zeta_{q}(2)$ sont linéairement indépendants sur $\mathbb{Q}$ pour $1 / q \in \mathbb{N} \backslash\{1\}$. Dans cette direction, le résultat principal de Krattenthaler, Rivoal et Zudilin dans [12] affirme que pour $1 / q \in \mathbb{Z} \backslash\{-1 ; 1\}$ et $A$ entier pair :

$$
\operatorname{dim}_{\mathbb{Q}}\left(\mathbb{Q}+\mathbb{Q} \zeta_{q}(3)+\cdots+\mathbb{Q} \zeta_{q}(A-1)\right) \geq f(A),
$$

où

$$
f(A)=\max _{\substack{r \in \mathbb{N} \\ 1 \leq r \leq A / 2}} f(r ; A) \quad \text { avec } f(r ; A):=\frac{4 r A+A-4 r^{2}}{\left(\frac{24}{\pi^{2}}+2\right) A+8 r^{2}} .
$$

Cette minoration donne des informations asymptotiques via l'équivalent

$$
f(A) \sim \frac{\pi}{2 \sqrt{\pi^{2}+12}} \sqrt{A} \text { lorsque } A \rightarrow+\infty,
$$

mais aussi quantitatives. En effet, il suffit de choisir une valeur de $A \geq 4$ la plus petite possible et donnant une dimension supérieure ou égale à 2 (l'idéal serait $A=4$, ce qui montrerait l'irrationalité de $\zeta_{q}(3)$ ). Cependant, il s'avère dans [12] que la valeur minimale exploitable est $A=12$, ce qui fournit le résultat suivant : pour $1 / q \in \mathbb{Z} \backslash\{-1 ; 1\}$, au moins l'un des nombres $\zeta_{q}(3), \zeta_{q}(5), \zeta_{q}(7), \zeta_{q}(9), \zeta_{q}(11)$ est irrationnel.

Le but de cet article est d'améliorer (1.1) et de raffiner le résultat quantitatif ci-dessus, en prouvant les deux théorèmes suivants.

Théorème 1.1. Pour $1 / q \in \mathbb{Z} \backslash\{-1 ; 1\}$ et tout entier pair $A \geq 4$, on a la minoration :

$$
\operatorname{dim}_{\mathbb{Q}}\left(\mathbb{Q}+\mathbb{Q} \zeta_{q}(3)+\cdots+\mathbb{Q} \zeta_{q}(A-1)\right) \geq g(A),
$$

où

$$
g(A)=\max _{\substack{r \in \mathbb{N} \\ 1 \leq r \leq A / 2}} g(r ; A) \quad \text { avec } g(r ; A):=\frac{4 r A+A-4 r^{2}}{\left(\frac{24}{\pi^{2}}+2\right) A-\frac{24}{\pi^{2}}+8 r^{2}},
$$


$g(A)$ vérifiant $g(A) \sim \frac{\pi}{2 \sqrt{\pi^{2}+12}} \sqrt{A}$ lorsque $A \rightarrow+\infty$.

On remarque ainsi qu'asymptotiquement, $g$ se comporte comme $f$ via l'égalité

$$
\lim _{A \rightarrow+\infty} \frac{g(A)}{\sqrt{A}}=\lim _{A \rightarrow+\infty} \frac{f(A)}{\sqrt{A}}=\frac{\pi}{2 \sqrt{\pi^{2}+12}} .
$$

Cependant, pour toute valeur fixée de $A$, ce premier théorème améliore la minoration de [12] puisque $g(A)>f(A)(\operatorname{car} g(r ; A)>f(r ; A))$. Cette comparaison donne en particulier les inégalités suivantes :

$$
\begin{aligned}
& f(10)<1<g(10)=g(10 ; 2) \simeq 1,001, \\
& f(38)<g(38)<2<f(40)<g(40), \\
& f(86)<3<g(86) .
\end{aligned}
$$

La conséquence immédiate de (1.3) est le Théorème 1.3 ci-dessous, qui est un raffinement de la version quantitative de [12] déjà mentionnée. Les inégalités (1.4) montrent que le Théorème 1.1 permet de retrouver, sans l'améliorer, le résultat suivant, déjà conséquence de (1.1) : pour $1 / q \in \mathbb{Z} \backslash\{-1 ; 1\}$, il existe deux entiers impairs $j_{1}$ et $j_{2}$ tels que $3 \leq j_{1}<j_{2} \leq 39$ et 1 , $\zeta_{q}\left(j_{1}\right)$ et $\zeta_{q}\left(j_{2}\right)$ soient linéairement indépendants sur $\mathbb{Q}$. En revanche, (1.5) fournit une amélioration par rapport à (1.1), qui peut s'écrire :

Corollaire 1.2. Pour $1 / q \in \mathbb{Z} \backslash\{-1 ; 1\}$, il existe trois entiers impairs $j_{1}$, $j_{2}$ et $j_{3}$ tels que $3 \leq j_{1}<j_{2}<j_{3} \leq 85$ et $1, \zeta_{q}\left(j_{1}\right), \zeta_{q}\left(j_{2}\right)$ et $\zeta_{q}\left(j_{3}\right)$ soient linéairement indépendants sur $\mathbb{Q}$.

Voici maintenant notre deuxième résultat, qui est une conséquence de (1.3) :

Théorème 1.3. Pour $1 / q \in \mathbb{Z} \backslash\{-1 ; 1\}$, au moins l'un des nombres $\zeta_{q}(3), \zeta_{q}(5)$, $\zeta_{q}(7), \zeta_{q}(9)$ est irrationnel.

Il est intéressant de noter que la technique adoptée dans [12 est tout à fait parallèle (mais dans le monde des $q$-analogues) à celle de [18], où il est démontré qu'au moins l'un des nombres $\zeta(5), \zeta(7), \ldots, \zeta(21)$ est irrationnel. Or les auteurs de [11] démontrent la conjecture des dénominateurs formulée dans [17], ce qui a pour conséquence le fait qu'au moins l'un des nombres $\zeta(5), \zeta(7), \ldots, \zeta(19)$ est irrationnel. C'est pourquoi le Théorème 1.3 n'est pas complètement une surprise, les auteurs de [12] estimant à la fin de l'introduction qu'il est 'probable' que l'on puisse prouver ce résultat, à condition de formuler et de démontrer une certaine ' $q$-conjecture des dénominateurs'.

Nous profitons de cette introduction pour donner les grandes lignes de démonstration du Théorème 1.1. Nous utilisons la proposition suivante, qui est un cas particulier du critère d'indépendance linéaire de Nesterenko [13] : 
Proposition 1.4. Soient un entier $N \geq 2$ et des réels $v_{1}, \ldots, v_{N}$. Supposons qu'il existe $N$ suites d'entiers $\left(p_{j, n}\right)_{n \geq 0}$ et des réels $\alpha_{1}$ et $\alpha_{2}$ avec $\alpha_{2}>0$ tels que :

i) $\lim _{n \rightarrow+\infty} \frac{1}{n^{2}} \log \left|p_{1, n} v_{1}+\cdots+p_{N, n} v_{N}\right|=-\alpha_{1}$,

ii) pour tout $j \in\{1, \ldots, N\}$, on a $\limsup _{n \rightarrow+\infty} \frac{1}{n^{2}} \log \left|p_{j, n}\right| \leq \alpha_{2}$.

Alors la dimension $d u \mathbb{Q}$-espace vectoriel engendré par $v_{1}, \ldots, v_{N}$ vérifie:

$$
\operatorname{dim}_{\mathbb{Q}}\left(\mathbb{Q} v_{1}+\cdots+\mathbb{Q} v_{N}\right) \geq 1+\frac{\alpha_{1}}{\alpha_{2}} .
$$

Remarque 1.5. Si en plus des hypothèses de ce critère on connait un facteur commun $\delta_{n}$ aux $p_{j, n}$, et si $\lim _{n \rightarrow+\infty} \frac{1}{n^{2}} \log \left|\delta_{n}\right|$ existe et vaut $\delta\left(<\alpha_{2}\right)$, alors en considérant les nouvelles suites d'entiers $\left(p_{j, n} / \delta_{n}\right)_{n \geq 0}$, on obtient :

$$
\operatorname{dim}_{\mathbb{Q}}\left(\mathbb{Q} v_{1}+\cdots+\mathbb{Q} v_{N}\right) \geq 1+\frac{\alpha_{1}+\delta}{\alpha_{2}-\delta} \quad\left(\geq 1+\frac{\alpha_{1}}{\alpha_{2}} \text { si } \quad \alpha_{1}>0\right) .
$$

Afin d'exploiter le critère de Nesterenko dans notre contexte, l'idée consiste à analyser la série hypergéométrique suivante (voir la partie 2 pour les notations) :

$$
\tilde{S}_{n}(q):=(q)_{n}^{A-2 r} \sum_{k \geq 1}\left(1-q^{2 k+n}\right) \frac{\left(q^{k-r n}, q^{k+n+1}\right)_{r n}}{\left(q^{k}\right)_{n+1}^{A}} q^{k(A-2 r) n / 2+k A / 2-k},
$$

où $|q| \neq 1, A$ entier, $r \in \mathbb{N}^{*}$ et $A>2 r$. Cette série a été suggérée, mais pas utilisée, dans [12], les auteurs préférant étudier une autre série, notée $S_{n}(q)$, pour prouver leurs résultats. La première étape est une réécriture de $\tilde{S}_{n}(q)$, sous forme d'une combinaison linéaire en des $\zeta_{q}(2 m+1), m \in \mathbb{N}^{*}$ :

$$
\tilde{S}_{n}(q)=\hat{P}_{0, n}(q)+\sum_{\substack{j=3 \\ j \text { impair }}}^{A-1} \hat{P}_{j, n}(q) \zeta_{q}(j)
$$

où $|q|<1, A$ est pair et les $\hat{P}_{j, n}(q)$ sont à priori dans $\mathbb{Q}(q)$, c'est-à dire des fractions rationnelles en $q$ (donc aussi en $1 / q$ ), à coefficients dans $\mathbb{Q}$. Dans un deuxième temps, on cherche un dénominateur commun $D_{n}(q)$ à ces fractions rationnelles en $1 / q$, vérifiant :

$$
D_{n}(q) \hat{P}_{j, n}(q) \in \mathbb{Z}\left[\frac{1}{q}\right] \quad \forall j \in\{0,3,5, \ldots, A-1\} .
$$

Lorsque $1 / q \in \mathbb{Z} \backslash\{-1 ; 1\}$, la Proposition 1.4 appliquée à la combinaison linéaire $D_{n}(q) \times \tilde{S}_{n}(q)$, ainsi que les estimations asymptotiques de $\tilde{S}_{n}(q)$, 
$\hat{P}_{j, n}(q)$ et $D_{n}(q)$, nous permettent de retrouver (1.1).

Notre amélioration, dont le résultat est donné par le Théorème 1.1, se situe au niveau du dénominateur commun $D_{n}(q)$ : nous formulons, puis démontrons, une $q$-conjecture des dénominateurs (voir le Théorème 4.1) qui fournit un nouveau dénominateur commun $\tilde{D}_{n}(q)$ divisant $D_{n}(q)$. La minoration (1.2) du Théorème 1.1 est obtenue via l'estimation asymptotique de $\delta_{n}:=D_{n}(q) / \tilde{D}_{n}(q)$ (voir la Remarque 1.5 qui suit la Propriété 1.4).

Remarque 1.6. Pour démontrer directement le Théorème 1.3, le critère de Nestenrenko n'est pas nécessaire. Il suffit en effet d'obtenir une estimation asymptotique de la combinaison linéaire à coefficients entiers $\tilde{D}_{n}(q) \times \tilde{S}_{n}(q)$, $1 / q \in \mathbb{Z} \backslash\{-1 ; 1\}$, en $\zeta_{q}(3), \zeta_{q}(5), \zeta_{q}(7)$ et $\zeta_{q}(9)$ (avec les choix $A=10$ et $r=2$ ). Il n'est donc pas nécessaire de borner la hauteur des coefficients de la combinaison linéaire, ceci n'est utile que pour l'indépendance linéaire.

Cet article est organisé comme suit. La deuxième partie est destinée à quelques notations concernant les $q$-séries qui seront utiles ensuite. La troisième partie est consacrée à l'étude de la série $\tilde{S}_{n}(q)$ évoquée ci-dessus. L'utilisation de $\tilde{S}_{n}(q)$ nous permet de redémontrer la minoration (1.1) de [12], et de dégager quelques lemmes clés. Dans cette même partie, nous expliquons par ailleurs comment le nouveau dénominateur $\tilde{D}_{n}(q)$ permet d'obtenir le Théorème 1.1. Dans la quatrième et dernière partie, nous nous consacrons exclusivement à l'étude de $\tilde{D}_{n}(q)$ : nous exprimons notre $q$-conjecture des dénominateurs (Théorème 4.1), et nous en donnons une démonstration utilisant une formule de transformation de séries hypergéométriques basiques due à Andrews [1, 2].

\section{Notations}

Donnons comme annoncé ci-dessus quelques définitions et notations issues du langage des $q$-séries, que le lecteur pourra retouver plus en détails dans [9].

Etant donné un nombre complexe $q$ (la "base") tel que $|q| \neq 1$, on définit pour tout réel $a$ et tout entier $k \in \mathbb{N}$, le $q$-factoriel montant par :

$$
(a)_{k} \equiv(a ; q)_{k}:=\left\{\begin{array}{l}
1 \text { si } k=0 \\
(1-a) \ldots\left(1-a q^{k-1}\right) \text { si } k>0 .
\end{array}\right.
$$

La base $q$ peut être omise lorsqu'il n'y a pas de confusion (en notant $(a)_{k}$ pour $(a ; q)_{k}$, etc), tout changement de base (par exemple $q$ remplacé par $p=$ $1 / q$ ) sera précisé dans les paragraphes concernés. Pour des raisons pratiques, notons pour $k \in \mathbb{N}$ :

$$
\left(a_{1}, \ldots, a_{m}\right)_{k}:=\left(a_{1}\right)_{k} \times \cdots \times\left(a_{m}\right)_{k} .
$$


Rappelons aussi le coefficient q-binomial :

$$
\left[\begin{array}{l}
n \\
k
\end{array}\right]_{q}=\left[\begin{array}{c}
n \\
k, n-k
\end{array}\right]_{q}:=\frac{(q)_{n}}{(q)_{k}(q)_{n-k}}
$$

et plus généralement le coefficient q-multinomial :

$$
\left[\begin{array}{c}
n \\
k_{1}, \ldots, k_{l}, n-k_{1}-\cdots-k_{l}
\end{array}\right]_{q}:=\frac{(q)_{n}}{(q)_{k_{1}} \ldots(q)_{k_{l}}(q)_{n-k_{1}-\cdots-k_{l}}},
$$

qui sont des polynômes en $q$, à coefficients entiers (voir par exemple [20]).

Enfin, rappelons la notion de série hypergéométrique basique ${ }_{s+1} \phi_{s}$ :

$$
{ }_{s+1} \phi_{s}\left[\begin{array}{c}
a_{0}, a_{1}, \ldots, a_{s} \\
b_{1}, \ldots, b_{s}
\end{array} ; q, z\right]:=\sum_{k=0}^{\infty} \frac{\left(a_{0}, a_{1}, \ldots, a_{s}\right)_{k}}{\left(q, b_{1}, \ldots, b_{s}\right)_{k}} z^{k},
$$

avec $a_{j} \in \mathbb{C}$ pour $0 \leq j \leq s$, et $b_{j} q^{k} \neq 1$ pour tout $k \in \mathbb{N}$ et $1 \leq j \leq s$. La série converge toujours pour $|z|<1$, et on dit que ${ }_{s+1} \phi_{s}$ est :

- bien équilibrée (well poised) si $q a_{0}=a_{1} b_{1}=\cdots=a_{s} b_{s}$

- très bien équilibrée (very well poised) si elle est bien équilibrée et de plus $a_{1}=q \sqrt{a_{0}}$.

\section{Une série très bien équilibrée}

Reprenons la série définie dans l'introduction par :

$$
\tilde{S}_{n}(q):=(q)_{n}^{A-2 r} \sum_{k \geq 1}\left(1-q^{2 k+n}\right) \frac{\left(q^{k-r n}, q^{k+n+1}\right)_{r n}}{\left(q^{k}\right)_{n+1}^{A}} q^{k(A-2 r) n / 2+k A / 2-k},
$$

pour $A$ entier pair, $r \in \mathbb{N}^{*}$ et $A-2 r>0$. Remarquons que cette série converge alors pour $|q| \neq 1$. La série $\tilde{S}_{n}(q)$ vérifie

$$
\tilde{S}_{n}(1 / q)=-q^{n(r-1)} \tilde{S}_{n}(q),
$$

relation qui provient du choix de la puissance de $q$ dans le sommande de (3.1). La relation (3.2) va permettre d'exprimer $\tilde{S}_{n}(q)$ comme combinaison linéaire sur $\mathbb{Q}$ des valeurs de $\zeta_{q}$ aux entiers impairs positifs seulement, alors que l'on pourrait s'attendre à priori à voir apparaître aussi les valeurs de $\zeta_{q}$ aux entiers pairs positifs. D'autre part on peut écrire

$$
\begin{aligned}
& \tilde{S}_{n}(q)=q^{(r n+1)((A-2 r) n / 2+A / 2-1)}\left(1-q^{n+2 r n+2}\right)(q)_{n}^{A-2 r} \frac{\left(q, q^{n+r n+2}\right)_{r n}}{\left(q^{r n+1}\right)_{n+1}^{A}} \\
& \times{ }_{A+4} \phi_{A+3}\left[\begin{array}{c}
a, q \sqrt{a},-q \sqrt{a}, q^{r n+1}, \ldots, q^{r n+1} \\
\sqrt{a},-\sqrt{a}, q^{(r+1) n+2}, \ldots, q^{(r+1) n+2} ; q, q^{(A-2 r) n / 2+A / 2-1}
\end{array}\right],
\end{aligned}
$$


avec $a=q^{(2 r+1) n+2}$, ce qui montre que $\tilde{S}_{n}(q)$ est une série hypergéométrique basique très bien équilibrée. Cette propriété nous permettra de formuler puis de démontrer notre $q$-conjecture des dénominateurs dans la partie 4 (voir le Théorème 4.1).

Pour $|q|<1, A$ entier pair et $r \in \mathbb{N}^{*}$ tel que $A-2 r>0$, nous allons successivement dans ce paragraphe démontrer les :

- Lemme 3.2 (paragraphe 3.1) : on a

$$
\tilde{S}_{n}(q)=\hat{P}_{0, n}(q)+\sum_{\substack{j=3 \\ j \text { impair }}}^{A-1} \hat{P}_{j, n}(q) \zeta_{q}(j)
$$

où pour $j \in\{0,3,5, \ldots, A-1\}$, les $\hat{P}_{j, n}(q)$ sont des fractions rationnelles en $q$ qui seront explicitées.

- Lemme 3.5 (paragraphe 3.2) : si on pose $d_{n}(q)=\operatorname{ppcm}\left(q-1, \ldots, q^{n}-1\right)$, alors pour $j \in\{0,3,5, \ldots, A-1\}$ et $\alpha=-A / 8-r^{2} / 2$, il existe des réels $\beta$ et $\gamma$ ne dépendant que de $A$ et $r$ tels que

$$
D_{n}(q) \hat{P}_{j, n}(q) \in \mathbb{Z}\left[\frac{1}{q}\right], \text { avec } D_{n}(q)=(A-1) ! q^{\left\lfloor\alpha n^{2}+\beta n+\gamma\right\rfloor} d_{n}(1 / q)^{A},
$$

où $\lfloor x\rfloor$ désigne la partie entière de $x$.

- Lemme 3.6 (paragraphe 3.3) : on a

$$
\lim _{n \rightarrow+\infty} \frac{1}{n^{2}} \log \left|\tilde{S}_{n}(q)\right|=-\frac{1}{2} r(A-2 r) \log |1 / q| .
$$

- Lemme 3.7 (paragraphe 3.3) : on a

$$
\limsup _{n \rightarrow+\infty} \frac{1}{n^{2}} \log \left|\hat{P}_{j, n}(q)\right| \leq \frac{1}{8}\left(A+4 r^{2}\right) \log |1 / q|, \quad \forall j \in\{0,3,5, \ldots, A-1\} .
$$

- Lemme 3.8 (paragraphe 3.3) : on a

$$
\lim _{n \rightarrow+\infty} \frac{1}{n^{2}} \log \left|D_{n}(q)\right|=\left(\frac{A}{8}+\frac{r^{2}}{2}+\frac{3 A}{\pi^{2}}\right) \log |1 / q|
$$

Alors ces cinq lemmes permettent, pour $1 / q \in \mathbb{Z} \backslash\{-1 ; 1\}$, d'appliquer la Proposition 1.4 à la combinaison linéaire

$$
D_{n}(q) \times \tilde{S}_{n}(q)=D_{n}(q) \hat{P}_{0, n}(q)+\sum_{\substack{j=3 \\ j \text { impair }}}^{A-1} D_{n}(q) \hat{P}_{j, n}(q) \zeta_{q}(j)
$$


avec les valeurs

$$
\alpha_{1}=-\left(\frac{A}{8}+\frac{r^{2}}{2}+\frac{3 A}{\pi^{2}}-\frac{r}{2}(A-2 r)\right) \log |1 / q|
$$

et

$$
\alpha_{2}=\left(\frac{A}{8}+\frac{r^{2}}{2}+\frac{3 A}{\pi^{2}}+\frac{A}{8}+\frac{r^{2}}{2}\right) \log |1 / q|=\left(\frac{A}{4}+r^{2}+\frac{3 A}{\pi^{2}}\right) \log |1 / q|,
$$

ce qui implique

$$
\begin{aligned}
\operatorname{dim}_{\mathbb{Q}}\left(\mathbb{Q}+\mathbb{Q} \zeta_{q}(3)+\mathbb{Q} \zeta_{q}(5)+\cdots+\mathbb{Q} \zeta_{q}(A-1)\right) & \\
& \geq 1+\frac{\alpha_{1}}{\alpha_{2}}=\frac{4 r A+A-4 r^{2}}{\left(\frac{24}{\pi^{2}}+2\right) A+8 r^{2}},
\end{aligned}
$$

redémontrant ainsi la minoration (1.1).

Cependant, des calculs numériques avec le logiciel Maple confirment (comme le fait que $\tilde{S}_{n}(q)$ soit très bien équilibrée le laissait espérer) que le dénominateur commun des $\hat{P}_{j, n}(q)$ du Lemme 3.2 pourrait bien être de la forme :

$$
\tilde{D}_{n}(q)=(A-1) ! q^{\left\lfloor\alpha n^{2}+\beta n+\gamma\right\rfloor} d_{n}(1 / q)^{A-1}, \quad \alpha=-\frac{A}{8}-\frac{r^{2}}{2},
$$

c'est-à-dire que l'on gagnerait une puissance de $d_{n}(1 / q)$ par rapport au choix $D_{n}(q)$. Ceci donnerait alors (voir la Remarque 1.5 qui suit la proposition 1.4, avec $\delta=\lim _{n \rightarrow \infty} \frac{1}{n^{2}} \log d_{n}(1 / q)$ et l'estimation (3.29) de $\left.d_{n}(1 / q)\right)$ :

$$
\begin{aligned}
\operatorname{dim}_{\mathbb{Q}}\left(\mathbb{Q}+\mathbb{Q} \zeta_{q}(3)+\mathbb{Q} \zeta_{q}(5)+\cdots\right. & \left.+\mathbb{Q} \zeta_{q}(A-1)\right) \\
& \geq 1+\frac{\alpha_{1}+\delta}{\alpha_{2}-\delta}=\frac{4 r A+A-4 r^{2}}{\left(\frac{24}{\pi^{2}}+2\right) A-\frac{24}{\pi^{2}}+8 r^{2}},
\end{aligned}
$$

ce qui démontrerait le Théorème 1.1. Nous en déduisons donc que pour prouver le Théorème 1.1, il nous suffit de montrer que le choix (3.3) est valide, c'est-à-dire

$$
\tilde{D}_{n}(q) \hat{P}_{j, n}(q) \in \mathbb{Z}\left[\frac{1}{q}\right] \quad \forall j \in\{0,3,5, \ldots, A-1\},
$$

ce qui fera l'objet de la quatrième partie. 


\subsection{Combinaisons linéaires en les $\zeta_{q}(2 j+1), j \in \mathbb{N}^{*}$}

Posons

$$
\tilde{R}_{n}(T ; q):=T^{(A-2 r) n / 2+A / 2-2} q^{-A n(n+1) / 2} \frac{(q)_{n}^{A-2 r}\left(q^{-r n} T, q^{n+1} T\right)_{r n}}{(T-1)^{A} \ldots\left(T-q^{-n}\right)^{A}},
$$

de sorte que

$$
\tilde{S}_{n}(q)=\sum_{k \geq 1} q^{k}\left(1-q^{2 k+n}\right) \tilde{R}_{n}\left(q^{k} ; q\right) .
$$

Remarquons que le degré en $T$ de la fraction rationnelle $\tilde{R}_{n}(T ; q)$ vaut $-(n+$ 1) $(A-2 r) / 2-r-2$ et est inférieur ou égal à -3 puisque $A>2 r \geq 2$. La décomposition de cette fraction en éléments simples s'écrit

$$
\tilde{R}_{n}(T ; q)=\sum_{s=1}^{A} \sum_{j=0}^{n} \frac{\tilde{c}_{s, j, n}(q)}{\left(T-q^{-j}\right)^{s}}=\sum_{s=1}^{A} \sum_{j=0}^{n} \frac{\tilde{d}_{s, j, n}(q)}{\left(1-T q^{j}\right)^{s}}
$$

avec $\tilde{d}_{s, j, n}(q)=(-1)^{s} q^{j s} \tilde{c}_{s, j, n}(q)$ et

$$
\begin{aligned}
\tilde{c}_{s, j, n}(q) & =\frac{1}{(A-s) !}\left[\frac{d^{A-s}}{d T^{A-s}} \tilde{R}_{n}(T ; q)\left(T-q^{-j}\right)^{A}\right]_{T=q^{-j}} \\
& =\frac{q^{-j s}}{(A-s) !}\left[\frac{d^{A-s}}{d u^{A-s}} \tilde{R}_{n}\left(u q^{-j} ; q\right)(u-1)^{A}\right]_{u=1} .
\end{aligned}
$$

La définition (3.5) conduit à la relation $\tilde{R}_{n}\left(T q^{n} ; 1 / q\right)=q^{n(r-2)} \tilde{R}_{n}(T ; q)$, qui a pour conséquence pour tous $j \in\{0, \ldots, n\}$ et $s \in\{1, \ldots, A\}$

$$
\tilde{d}_{s, n-j, n}(1 / q)=q^{n(r-2)} \tilde{d}_{s, j, n}(q),
$$

ou de façon équivalente

$$
\tilde{c}_{s, n-j, n}(1 / q)=q^{n(s+r-2)} \tilde{c}_{s, j, n}(q) .
$$

Remarque 3.1. La relation (3.8) est un peu différente de celle prouvée dans [12], sauf dans le cas $r=1$, ce qui n'est pas une surprise car la série $\tilde{S}_{n}(q)$ coincide pour $r=1$ avec la série $S_{n}^{[1]}(q):=S_{n}(q)-S_{n}(1 / q)$ utilisée dans [12].

Nous aurons besoin dans ce qui suit des nombres de Stirling de première espèce sans signe (voir [20]), qui sont des nombres entiers notés $c(s, j)$ (où $s$ et $j$ sont deux entiers tels que $1 \leq j \leq s$ ) et définis par

$$
x(x+1) \ldots(x+s-1)=\sum_{j=1}^{s} c(s, j) x^{j} .
$$


Lemme 3.2. On a pour $|q|<1$, A pair et $r \in \mathbb{N}^{*}$ tel que $A-2 r>0$ :

$$
\tilde{S}_{n}(q)=\hat{P}_{0, n}(q)+\sum_{\substack{j=3 \\ j \text { impair }}}^{A-1} \hat{P}_{j, n}(q) \zeta_{q}(j)
$$

où pour $j=3,5, \ldots, A-1$,

$$
\begin{aligned}
\hat{P}_{0, n}(q) & :=\tilde{P}_{0, n}(1, q)-q^{-n(r-1)} \tilde{P}_{0, n}(1,1 / q)-\left[\frac{d}{d z} \tilde{P}_{1, n}(z, q)\right]_{z=1}, \\
\hat{P}_{j, n}(q) & :=\sum_{s=j}^{A} \frac{2 c(s-1, j-1)}{(s-1) !} \tilde{P}_{s, n}(1, q)
\end{aligned}
$$

et

$$
\begin{aligned}
& \tilde{P}_{0, n}(z, q):=\sum_{s=1}^{A} \sum_{j=1}^{n} \sum_{k=1}^{j}(-1)^{s} \frac{q^{k-j(1-s)}}{\left(1-q^{k}\right)^{s}} \tilde{c}_{s, j, n}(q) z^{j-k}, \\
& \tilde{P}_{s, n}(z, q):=(-1)^{s} \sum_{j=0}^{n} q^{j(s-1)} \tilde{c}_{s, j, n}(q) z^{j} .
\end{aligned}
$$

Démonstration. Reprenons les fonctions intermédiaires de [12] définies par $Z_{s}(z ; q):=\sum_{k \geq 1} \frac{q^{k}}{\left(1-q^{k}\right)^{s}} z^{-k}$ et $Z_{s}(q):=Z_{s}(1 ; q)$, vérifiant notamment pour $s \geq 2$

$$
Z_{s}(q)-Z_{s}(1 / q)=\frac{2}{(s-1) !} \sum_{\substack{j=3 \\ j \text { impair }}}^{s} c(s-1, j-1) \zeta_{q}(j),
$$

et convergeant pour tout $s \geq 1$ dès que $|q|<|z|$. Nous utiliserons le fait que $Z_{s}(1 / z ; 1 / q)$ converge pour tout $|z|<|q|^{1-s}$. Posons alors pour $|q|<|z|$

$$
\mathcal{S}_{n}(z ; q):=\sum_{k \geq 1} q^{k} \tilde{R}_{n}\left(q^{k} ; q\right) z^{-k}
$$

Il n'est pas difficile de voir que $\mathcal{S}_{n}(z ; q)$ converge dès que $|z|>|q|^{(A-2 r) n / 2+A / 2-1}$ et $\mathcal{S}_{n}(1 / z ; 1 / q)$ converge dès que $|z|<|q|^{-(A-2 r) n / 2-A / 2-1}$. Les deux séries $\mathcal{S}_{n}(z ; q)$ et $\mathcal{S}_{n}(1 / z ; 1 / q)$ convergent donc simultanément pour $|q|<|z| \leq 1$ lorsque $A \geq 4$. Un calcul simple montre que

$$
\mathcal{S}_{n}(1 ; q)-q^{-n(r-1)} \mathcal{S}_{n}(1 ; 1 / q)=\tilde{S}_{n}(q) .
$$

Par ailleurs, en utilisant la décomposition en éléments simples de $\tilde{R}_{n}(T ; q)$, on obtient

$$
\mathcal{S}_{n}(z ; q)=\tilde{P}_{0, n}(z, q)+\sum_{s=1}^{A} \tilde{P}_{s, n}(z, q) Z_{s}(z ; q),
$$


où $\tilde{P}_{0, n}(z, q)$ et $\tilde{P}_{s, n}(z, q)$ sont des polynômes en $z$ définis par (3.13) et (3.14). Or, en reprenant la définition (3.14), on s'aperçoit que la relation (3.8) se traduit pour $s \geq 1$ par $\tilde{P}_{s, n}(1 / z, 1 / q)=z^{-n} q^{n(r-1)} \tilde{P}_{s, n}(z, q)$, ce qui nous donne l'idée d'étudier maintenant la série

$$
\tilde{\mathcal{S}}_{n}(z ; q):=\mathcal{S}_{n}(z ; q)-z^{n} q^{-n(r-1)} \mathcal{S}_{n}(1 / z ; 1 / q),
$$

avec la condition $|q|<|z|<1$ assurant la convergence. Le développement (3.17), puis la relation ci-dessus entre $\tilde{P}_{s, n}(1 / z, 1 / q)$ et $\tilde{P}_{s, n}(z, q)$, permettent alors d'écrire pour $|q|<|z|<1$

$$
\begin{aligned}
\tilde{\mathcal{S}}_{n}(z ; q)=\tilde{P}_{0, n}(z, q)-q^{-n(r-1)} z^{-n} \tilde{P}_{0, n}(1 / z, 1 / q) & \\
& +\sum_{s=1}^{A} \tilde{P}_{s, n}(z, q)\left(Z_{s}(z ; q)-Z_{s}(1 / z ; 1 / q)\right) .
\end{aligned}
$$

Il ne reste plus qu'à faire tendre $z$ vers 1 dans (3.19) pour obtenir le Lemme 3.2, via la définition (3.18) et les égalités (3.15) et (3.16), étant entendu (comme dans [12]) que

$$
\lim _{z \rightarrow 1} \tilde{P}_{1, n}(z, q)\left(Z_{1}(z ; q)-Z_{1}(1 / z ; 1 / q)\right)=-\left[\frac{d}{d z} \tilde{P}_{1, n}(z, q)\right]_{z=1} .
$$

\subsection{Propriétés arithmétiques des $\hat{P}_{j, n}$}

En vue de trouver un dénominateur commun $D_{n}(q)$ aux coefficients $\hat{P}_{j, n}(q) \in$ $\mathbb{Q}(q)$, rappelons que $d_{n}(q) \in \mathbb{Z}[q]$ est le polynôme unitaire, de plus petit degré et multiple commun de $1-q, 1-q^{2}, \ldots, 1-q^{n}$. On démontre alors les deux lemmes suivants, qui permettront de déduire le Lemme 3.5.

Lemme 3.3. Pour tous $s \in\{1, \ldots, A\}$ et $j \in\{0, \ldots, n\}$, on $a$ :

$$
d_{n}(1 / q)^{A-s} \tilde{c}_{s, j, n}(q) \in \mathbb{Z}\left[q ; \frac{1}{q}\right]
$$

Démonstration. Écrivons (3.6) sous la forme

$$
\tilde{c}_{s, j, n}(q)=\frac{1}{(A-s) !}\left[\frac{d^{A-s}}{d T^{A-s}} \tilde{V}_{n}(T ; q)\right]_{T=q^{-j}},
$$

avec

$$
\begin{aligned}
\tilde{V}_{n}(T ; q):= & \tilde{R}_{n}(T ; q)\left(T-q^{-j}\right)^{A} \\
= & (q)_{n}^{A-2 r} T^{(A-2 r) n / 2+A / 2-2} q^{-A n(n+1) / 2} \\
& \quad \times \frac{\left(q^{-r n} T, q^{n+1} T\right)_{r n}}{(T-1)^{A} \ldots\left(T-q^{-n}\right)^{A}}\left(T-q^{-j}\right)^{A}
\end{aligned}
$$


On regroupe les termes de $\tilde{V}_{n}(T ; q)$ de la manière suivante :

$$
\tilde{V}_{n}(T ; q)=q^{a n^{2}+b n+c} T^{A / 2-2} F(T)^{A / 2-r} G(T)^{A / 2-r} \prod_{l=1}^{r} H_{l}(T) I_{l}(T),
$$

où $a, b$ et $c$ sont des entiers dépendant uniquement de $A$ et $r$, les fonctions $F, G, H_{l}$ et $I_{l}$ étant celles définies dans [12. Donc en utilisant leurs décompositions en éléments simples (voir [12]), on s'aperçoit que si l'on note $U$ n'importe laquelle de ces fonctions, ou même $T \mapsto T^{A / 2-2}$, alors

$$
\frac{d_{n}(1 / q)^{\mu}}{\mu !}\left[\frac{d^{\mu}}{d T^{\mu}} U(T)\right]_{T=q^{-j}} \in \mathbb{Z}\left[q ; \frac{1}{q}\right] \quad \forall \mu \in \mathbb{N} .
$$

On conclut en utilisant (3.20) et en appliquant la formule de Leibniz de dérivation $(\mu=A-s)$-ième d'un produit de fonctions.

Lemme 3.4. Soit $\alpha=-A / 8-r^{2} / 2$. Il existe alors $\beta^{\prime}$ et $\gamma^{\prime}$ réels dépendant uniquement de $A$ et $r$ tels que pour tous $(s, j) \in\{1, \ldots, A\} \times\{0, \ldots, n\}$ :

$$
\lim _{q \rightarrow+\infty} q^{\alpha n^{2}+\beta^{\prime} n+\gamma^{\prime}} \tilde{c}_{s, j, n}(q)<\infty .
$$

Démonstration. Reprenons l'expression (3.20) de la démonstration précédente, et posons $\tilde{v}_{n}(T ; q):=\frac{d}{d T} \tilde{V}_{n}(T ; q) / \tilde{V}_{n}(T ; q)$ la dérivée logarithmique en $T$ de $\tilde{V}_{n}(T ; q)$. Comme dans [12], la formule de dérivation de Faà di Bruno donne alors pour tout $\mu \in \mathbb{N}$

$$
\frac{1}{\mu !} \frac{d^{\mu}}{d T^{\mu}} \tilde{V}_{n}(T ; q)=\sum_{k_{1}+\cdots+\mu k_{\mu}=\mu} \frac{\tilde{V}_{n}(T ; q)}{k_{1} ! \ldots k_{\mu} !} \prod_{l=1}^{\mu}\left(\frac{1}{l !} \frac{d^{l-1}}{d T^{l-1}} \tilde{v}_{n}(T ; q)\right)^{k_{l}} .
$$

Or par définition

$$
\begin{aligned}
\tilde{v}_{n}(T ; q) & =\frac{d}{d T}\left(\log \tilde{V}_{n}(T ; q)\right) \\
= & \frac{(A-2 r) n / 2+A / 2-2}{T}+\sum_{i=1}^{r n} \frac{1}{T-q^{i}} \\
& \quad+\sum_{i=n+1}^{r n+n} \frac{1}{T-q^{-i}}-A \sum_{\substack{i=0 \\
i \neq j}}^{n} \frac{1}{T-q^{-i}},
\end{aligned}
$$


donc pour $l \in \mathbb{N}$

$$
\begin{aligned}
\frac{(-1)^{l-1}}{(l-1) !} \frac{d^{l-1}}{d T^{l-1}} \tilde{v}_{n}(T ; q)=\frac{(A-2 r) n / 2+A / 2-2}{T^{l}}+\sum_{i=1}^{r n} \frac{1}{\left(T-q^{i}\right)^{l}} \\
+\sum_{i=n+1}^{r n+n} \frac{1}{\left(T-q^{-i}\right)^{l}}-A \sum_{\substack{i=0 \\
i \neq j}}^{n} \frac{1}{\left(T-q^{-i}\right)^{l}}
\end{aligned}
$$

Or on peut écrire ceci sous la forme

$$
\begin{gathered}
\frac{(-1)^{l-1}}{(l-1) !} \frac{d^{l-1}}{d T^{l-1}} \tilde{v}_{n}(T ; q)=\frac{(A-2 r) n / 2+A / 2-2}{T^{l}}+\sum_{i=1}^{r n}\left(\frac{q^{-i}}{T q^{-i}-1}\right)^{l} \\
+\sum_{i=n+1}^{r n+n} \frac{1}{\left(T-q^{-i}\right)^{l}}-A \sum_{i=0}^{j-1}\left(\frac{q^{i}}{T q^{i}-1}\right)^{l}-A \sum_{i=j+1}^{n} \frac{1}{\left(T-q^{-i}\right)^{l}}
\end{gathered}
$$

ce qui implique que $\forall j \in\{0, \ldots, n\}, \lim _{q \rightarrow+\infty} q^{-j l}\left[\frac{d^{l-1}}{d T^{l-1}} \tilde{v}_{n}(T ; q)\right]_{T=q^{-j}}<\infty$. On en déduit que pour $k_{1}+\cdots+\mu k_{\mu}=\mu$,

$$
\lim _{q \rightarrow+\infty} q^{-j \mu}\left[\prod_{l=1}^{\mu}\left(\frac{1}{l !} \frac{d^{l-1}}{d T^{l-1}} \tilde{v}_{n}(T ; q)\right)^{k_{l}}\right]_{T=q^{-j}}<\infty .
$$

Par ailleurs la puissance dominante de $q$ apparaissant dans $\tilde{V}_{n}\left(q^{-j} ; q\right)$ défini grâce à (3.21) est de la forme :

$$
j(n A / 2-A+2)-j^{2} A / 2+r n(r n-1) / 2 .
$$

Il suffit maintenant de choisir $\mu=A-s$ dans (3.22), puis à l'aide de (3.20) et de (3.23) on obtient :

$$
\lim _{q \rightarrow+\infty} q^{-j(A-s)-\left(j(n A / 2-A+2)-j^{2} A / 2+r n(r n-1) / 2\right)} \tilde{c}_{s, j, n}(q)<\infty .
$$

On conclut alors aisément puisque pour tous $(s, j) \in\{1, \ldots, A\} \times\{0, \ldots, n\}$,

$$
-j(A-s)-\left(j(n A / 2-A+2)-j^{2} A / 2+r n(r n-1) / 2\right) \geq \alpha n^{2}+\beta^{\prime} n+\gamma^{\prime},
$$

avec $\alpha=-A / 8-r^{2} / 2, \beta^{\prime}=(r-1) / 2, \gamma^{\prime}=-1 /(2 A)$ (cette borne inférieure est obtenue pour $s=1$ et $j=n / 2+1 / A)$. 
Le Lemme 3.4 implique que $\lim _{q \rightarrow+\infty} q^{\alpha n^{2}+\beta^{\prime} n+\gamma^{\prime}} d_{n}(1 / q)^{A-s} \tilde{c}_{s, j, n}(q)<\infty$ pour tous $(s, j) \in\{1, \ldots, A\} \times\{0, \ldots, n\}$ puisque $\lim _{q \rightarrow+\infty} d_{n}(1 / q)=d_{n}(0)=$ \pm 1 . En utilisant le Lemme 3.3 on obtient donc que pour $\alpha=-A / 8-r^{2} / 2$ il existe $\beta^{\prime}$ et $\gamma^{\prime}$ réels ne dépendant que de $A$ et $r$, tels que pour tous $(s, j) \in$ $\{1, \ldots, A\} \times\{0, \ldots, n\}$

$$
q^{\left\lfloor\alpha n^{2}+\beta^{\prime} n+\gamma^{\prime}\right\rfloor} d_{n}(1 / q)^{A-s} \tilde{c}_{s, j, n}(q) \in \mathbb{Z}\left[\frac{1}{q}\right] .
$$

D'après les expressions (3.11)-(3.14), l'équation (3.9), la définition de $d_{n}(1 / q)$ et le fait que

$$
\left[\frac{d}{d z} \tilde{P}_{1, n}(z, q)\right]_{z=1}=-\sum_{j=0}^{n} j \tilde{c}_{1, j, n}(q),
$$

on déduit de (3.24) le lemme suivant (de simples calculs montrent que les valeurs $\beta=\beta^{\prime}-A+1$ et $\gamma=\gamma^{\prime}+A-2$ conviennent) :

Lemme 3.5. Pour $\alpha=-A / 8-r^{2} / 2$, il existe $\beta$ et $\gamma$ réels ne dépendant que de $A$ et $r$ tels que:

$$
(A-1) ! q^{\left\lfloor\alpha n^{2}+\beta n+\gamma\right\rfloor} d_{n}(1 / q)^{A-j} \hat{P}_{j, n}(q) \in \mathbb{Z}\left[\frac{1}{q}\right] \quad \forall j \in\{3,5, \ldots, A-1\}
$$

et

$$
q^{\left\lfloor n^{2}+\beta n+\gamma\right\rfloor} d_{n}(1 / q)^{A} \hat{P}_{0, n}(q) \in \mathbb{Z}\left[\frac{1}{q}\right] .
$$

Ainsi, en posant

$$
D_{n}(q)=(A-1) ! q^{\left\lfloor\alpha n^{2}+\beta n+\gamma\right\rfloor} d_{n}(1 / q)^{A},
$$

on obtient :

$$
D_{n}(q) \hat{P}_{j, n}(q) \in \mathbb{Z}\left[\frac{1}{q}\right] \forall j \in\{0,3,5, \ldots, A-1\}
$$

\subsection{Estimations asymptotiques}

On évalue maintenant asymptotiquement $\tilde{S}_{n}(q)$, puis les coefficients $\hat{P}_{j, n}(q)$ de (3.10), et enfin $D_{n}(q)$. Fixons $A$ entier pair et $r \in \mathbb{N}^{*}$ tel que $A-2 r>0$.

Commençons par l'estimation asymptotique de $\tilde{S}_{n}(q)$, donnée par le lemme suivant :

Lemme 3.6. Pour tout $|q|<1$, on a :

$$
\lim _{n \rightarrow+\infty} \frac{1}{n^{2}} \log \left|\tilde{S}_{n}(q)\right|=-\frac{1}{2} r(A-2 r) \log |1 / q|
$$


Démonstration. On note $\rho_{k}(q)=q^{k}\left(1-q^{2 k+n}\right) \tilde{R}_{n}\left(q^{k} ; q\right)$, de sorte que $\tilde{S}_{n}(q)=$ $\sum_{k \geq 1} \rho_{k}(q)$. Par la définition (3.5) de $\tilde{R}_{n}(T ; q)$, il apparait clairement que $\rho_{k}(q)=0 \Leftrightarrow k \in\{0, \ldots, r n\}$. Or on a pour $k \geq r n+1$

$$
\frac{\rho_{k+1}(q)}{\rho_{k}(q)}=q^{(A-2 r) n / 2+A / 2-1} \frac{1-q^{2 k+n+2}}{1-q^{2 k+n}} \frac{1-q^{1+k+n+r n}}{1-q^{k-r n}}\left(\frac{1-q^{k}}{1-q^{k+n+1}}\right)^{A+1} \text {. }
$$

Comme $A-2 r>0,|q|<1$ et $k \geq r n+1$, on a donc pour $n$ grand la majoration uniforme en $k$ :

$$
\left|\frac{\rho_{k+1}(q)}{\rho_{k}(q)}\right| \leq|q|^{(A-2 r) n / 2}\left(\frac{1+|q|}{1-|q|}\right)^{A+3}<\frac{1}{3}
$$

ce qui permet comme dans [12] d'écrire l'encadrement

$$
\frac{1}{2}\left|\rho_{r n+1}(q)\right| \leq\left|\tilde{S}_{n}(q)\right| \leq \frac{3}{2}\left|\rho_{r n+1}(q)\right| .
$$

Or

$$
\rho_{r n+1}(q)=\left(1-q^{2 r n+n+2}\right)(q)_{n}^{A-2 r} \frac{\left(q, q^{(r+1) n+2}\right)_{r n}}{\left(q^{r n+1}\right)_{n+1}^{A}} q^{(r n+1)((A-2 r) n / 2+A / 2-1)},
$$

donc on obtient

$$
\lim _{n \rightarrow+\infty} \frac{1}{n^{2}} \log \left|\tilde{S}_{n}(q)\right|=\lim _{n \rightarrow+\infty} \frac{1}{n^{2}} \log \left|\rho_{r n+1}(q)\right|=-\frac{1}{2} r(A-2 r) \log |1 / q| .
$$

Donnons maintenant l'estimation asymptotique des coefficients $\hat{P}_{j, n}(q)$ de (3.10), à l'aide du lemme suivant :

Lemme 3.7. Pour tout $j \in\{0,3,5, \ldots, A-1\}$ et $|q|<1$, on a :

$$
\limsup _{n \rightarrow+\infty} \frac{1}{n^{2}} \log \left|\hat{P}_{j, n}(q)\right| \leq \frac{1}{8}\left(A+4 r^{2}\right) \log |1 / q| .
$$

Démonstration. Remarquons tout d'abord que pour des nombres complexes $a_{i, n}(0 \leq i \leq n)$

$$
\left(\forall i \in\{0, \ldots, n\}, \limsup _{n \rightarrow+\infty} \frac{1}{n^{2}} \log \left|a_{i, n}\right| \leq c\right) \Rightarrow \limsup _{n \rightarrow+\infty} \frac{1}{n^{2}} \log \left|\sum_{i=0}^{n} a_{i, n}\right| \leq c .
$$

Ceci montre, via les définitions des $\hat{P}_{j, n}(q)$ données par (3.11)-(3.14), qu'il suffit de prouver que l'estimation du lemme est valide pour les coefficients $\tilde{d}_{s, j, n}(q)=(-1)^{s} q^{j s} \tilde{c}_{s, j, n}(q)$, uniformément en $j$ et $s$. Fixons maintenant $j \in$ 
$\{0, \ldots, n\}$ et $\eta=(1-|q|) / 2>0$; la formule de Cauchy appliquée à (3.6) donne alors

$$
\tilde{d}_{s, j, n}(q)=-\frac{1}{2 i \pi} \int_{\mathcal{C}} \tilde{R}_{n}\left(T q^{-j} ; q\right)(1-T)^{s-1} d T,
$$

où $\mathcal{C}$ désigne le cercle de centre 1 et de rayon $\eta$. Reprenons donc l'expression (3.5), qui conduit à

$$
\begin{aligned}
\tilde{R}_{n}\left(T q^{-j} ; q\right)(1-T)^{s-1}=q^{-j((A-2 r) n / 2+A / 2-2)} T^{(A-2 r) n / 2+A / 2-2} & \\
& \times(q)_{n}^{A-2 r}(1-T)^{s-1} \frac{\left(q^{-r n-j} T, q^{n-j+1} T\right)_{r n}}{\left(T q^{-j}\right)_{n+1}^{A}} .
\end{aligned}
$$

Ceci peut s'écrire après quelques transformations élémentaires

$$
\begin{aligned}
\tilde{R}_{n}\left(T q^{-j} ; q\right)(1-T)^{s-1} & =q^{A j^{2} / 2-A n j / 2+2 j-r n(r n+1) / 2} T^{A(n-2 j) / 2+A / 2-2} \\
& \times(-1)^{A j+r n}(q)_{n}^{A-2 r}(1-T)^{s-A-1} \frac{\left(q^{j+1} / T, q^{n-j+1} T\right)_{r n}}{(q / T)_{j}^{A}(q T)_{n-j}^{A}} .
\end{aligned}
$$

En vue de majorer cette expression pour $T \in \mathcal{C}$, on reprend maintenant les encadrements de [12] valables pour $(a, b) \in \mathbb{N}^{*} \times \mathbb{N}, T \in \mathcal{C}$ et $\eta=(1-|q|) / 2$ :

$$
0<(|q|(1+\eta) ;|q|)_{\infty} \leq\left|\left(q^{a} T\right)_{b}\right| \leq(-(1+\eta) ;|q|)_{\infty}
$$

et

$$
0<(|q| /(1-\eta) ;|q|)_{\infty} \leq\left|\left(q^{a} / T\right)_{b}\right| \leq(-1 /(1-\eta) ;|q|)_{\infty}
$$

puis

$$
\left|T^{A(n-2 j) / 2+A / 2-2}\right| \leq\left(\max (1+\eta ; 1 /(1-\eta))^{A n / 2}(1+\eta)^{A / 2-2}\right.
$$

et

$$
\left|(q)_{n}\right| \leq(-|q| ;|q|)_{\infty} \text { et }|1-T|^{s-A-1} \leq 1 / \eta^{A+1}
$$

Il ne reste donc plus qu'à majorer la puissance de $q$ dans l'expression de $\tilde{R}_{n}\left(T q^{-j} ; q\right)(1-T)^{s-1}$ ci-dessus. Ceci se fait en remarquant que la fonction $j \mapsto A j^{2} / 2-A n j / 2+2 j-r n(r n+1) / 2$ atteint son maximum en $j=n / 2-2 / A$, et cette valeur maximale est de la forme $-A n^{2} / 8-r^{2} n^{2} / 2+\lambda n+\mu, \lambda$ et $\mu$ étant des réels ne dépendant que de $A$ et $r$. Tout cela conduit à la majoration

$$
\left|\tilde{d}_{s, j, n}(q)\right| \leq \tilde{c}_{0} \times|q|^{-\left(A+4 r^{2}\right) n^{2} / 8},
$$

où $\tilde{c}_{0}$ ne dépend ni de $j$ ni de $s$, et vérifie $\lim _{n \rightarrow+\infty} \tilde{c}_{0}^{1 / n^{2}}=1$, ce qui permet de conclure. 
Finalement, donnons l'estimation asymptotique de $D_{n}(q)$ défini en (3.28) :

Lemme 3.8. Pour tout $|q|<1$ on a

$$
\lim _{n \rightarrow+\infty} \frac{1}{n^{2}} \log \left|D_{n}(q)\right|=\left(\frac{A}{8}+\frac{r^{2}}{2}+\frac{3 A}{\pi^{2}}\right) \log |1 / q| .
$$

Démonstration. Pour $|q|<1$ on a l'estimation (voir [7] et [21])

$$
\lim _{n \rightarrow+\infty} \frac{1}{n^{2}} \log \left|d_{n}(1 / q)\right|=\frac{3}{\pi^{2}} \log |1 / q|
$$

donc la conclusion est immédiate à l'aide des expressions de $D_{n}(q)$ et de $\alpha$ données par (3.28).

\section{Démonstration du Théorème 1.1}

\subsection{La $q$-conjecture des dénominateurs}

D'après le Lemme 3.5, le dénominateur commun à tous les coefficients $\hat{P}_{j, n}(q)$ $(j \in\{0,3,5, \ldots, A-1\})$ dans l'expression (3.10) est de la forme $D_{n}(q)=(A-$ $1) ! q^{\left\lfloor\alpha n^{2}+\beta n+\gamma\right\rfloor} d_{n}(1 / q)^{A}$, avec $\alpha=-A / 8-r^{2} / 2$. Comme nous l'avons vu dans la partie précédente, l'amélioration donnée par le Théorème 1.1 correspond au gain d'une puissance de $d_{n}(1 / q)$ dans ce dénominateur commun. Or il apparait clairement dans (3.26) que le dénominateur commun aux $\hat{P}_{j, n}(q)$ pour $j \in\{3,5, \ldots, A-1\}$ est de la forme $\tilde{D}_{n}(q)=(A-1) ! q^{\left\lfloor\alpha n^{2}+\beta n+\gamma\right\rfloor} d_{n}(1 / q)^{A-1}$, avec $\alpha=-A / 8-r^{2} / 2$. Il suffit donc de prouver que cela reste valable pour $\hat{P}_{0, n}(q)$. Ainsi le Théorème 1.1 est une conséquence du résultat suivant, qui est l'expression de notre $q$-conjecture des dénominateurs :

Théorème 4.1. Soient $A$ entier pair et $r \in \mathbb{N}^{*}$ tel que $A-2 r>0$. Soit $\alpha=-A / 8-r^{2} / 2$; il existe $\beta$ et $\gamma$ réels ne dépendant que de $A$ et $r$ tels que:

$$
q^{\left\lfloor\alpha n^{2}+\beta n+\gamma\right\rfloor} d_{n}(1 / q)^{A-1} \hat{P}_{0, n}(q) \in \mathbb{Z}\left[\frac{1}{q}\right] .
$$

Remarque 4.2. Il est possible, mais inutile dans le cadre de la preuve du Théorème 1.1 à laquelle se limite cet article, de prouver avec des outils similaires à ceux qui suivent qu'en fait :

$$
(A-1) ! q^{\left\lfloor\alpha n^{2}+\beta n+\gamma\right\rfloor} d_{n}(1 / q)^{A-j-1} \hat{P}_{j, n}(q) \in \mathbb{Z}\left[\frac{1}{q}\right] \forall j \in\{0,3,5, \ldots, A-1\} .
$$




\subsection{Une condition suffisante}

En vertu du fait que $\lim _{q \rightarrow+\infty} d_{n}(1 / q)=d_{n}(0)= \pm 1$ et de la formule (3.27) du Lemme 3.5, il suffit pour prouver le Théorème 4.1 de montrer que

$$
d_{n}(1 / q)^{A-1} \hat{P}_{0, n}(q) \in \mathbb{Z}\left[q ; \frac{1}{q}\right] .
$$

D'abord, le troisième terme de (3.11) servant dans le Lemme 3.2 à définir $\hat{P}_{0, n}(q)$ vérifie grâce à (3.24) et (3.25) :

$$
d_{n}(1 / q)^{A-1}\left[\frac{d}{d z} \tilde{P}_{1, n}(z, q)\right]_{z=1} \in \mathbb{Z}\left[q ; \frac{1}{q}\right]
$$

Il suffit donc de démontrer que

$$
d_{n}(1 / q)^{A-1}\left(\tilde{P}_{0, n}(1, q)-q^{-n(r-1)} \tilde{P}_{0, n}(1,1 / q)\right) \in \mathbb{Z}\left[q ; \frac{1}{q}\right] .
$$

Reprenons donc la définition (3.13) de $\tilde{P}_{0, n}(z, q)$, qui à l'aide d'un calcul simple et de (3.9) permet d'écrire :

$$
\tilde{P}_{0, n}(1, q)-q^{-n(r-1)} \tilde{P}_{0, n}(1,1 / q)=\sum_{s=1}^{A} \sum_{k=1}^{n} \frac{1}{\left(1-q^{-k}\right)^{s}} V_{k},
$$

où

$$
V_{k}=\frac{1}{(A-s) !}\left[\frac{d^{A-s}}{d u^{A-s}} \sum_{j=k}^{n} q^{-k(s-1)} e_{j}(u)-(-1)^{s} q^{-k} e_{n-j}(u)\right]_{u=1}
$$

avec comme notation

$$
\begin{aligned}
e_{j}(u):= & q^{-j} \tilde{R}_{n}\left(q^{-j} u, q\right)(u-1)^{A} \\
= & (-1)^{r n} u^{A / 2-2+(n-2 j) A / 2} q^{-r^{2} n^{2} / 2-n r / 2-j(n-j) A / 2+j} \\
& \times\left(\frac{(q)_{r n}}{(q)_{n}^{r}}\right)^{2}\left(\frac{(q)_{n}}{\left(q u^{-1}\right)_{j}(q u)_{n-j}}\right)^{A} \frac{\left(q^{j+1} u^{-1}, q^{n+1-j} u\right)_{r n}}{(q, q)_{r n}}
\end{aligned}
$$

La formule (4.1) est en fait obtenue en inversant les sommes en $j$ et en $k$ définies en (3.13). Cette étape déterminante nous permettra d'extraire un facteur de $V_{k}$ (voir (4.5) ci-dessous). Notons que l'on a

$$
\tilde{c}_{s, j, n}(q)=\frac{1}{(A-s) !}\left[q^{-j(s-1)} \frac{d^{A-s}}{d u^{A-s}} e_{j}(u)\right]_{u=1},
$$


et que $e_{j}(u)$ est en fait défini pour $A \in 2 \mathbb{N}$ et $r \in \mathbb{N}$ quelconque.

Du Lemme 3.3 et de la relation (4.4), on déduit que pour tout $s \in$ $\{1, \ldots, A\}$ et $j \in\{1, \ldots, n\}, d_{n}(1 / q)^{A-s}\left[d^{A-s} e_{j}(u) / d u^{A-s}\right]_{u=1} \in \mathbb{Z}[q ; 1 / q]$, et par suite que pour tout $k \in\{1, \ldots, n\}, d_{n}(1 / q)^{A-s} V_{k} \in \mathbb{Z}[q ; 1 / q]$. Ceci ne prouve pas le Théorème 4.1, mais par définition de $d_{n}(q)$, il suffit via l'équation (4.1) de montrer que pour tous $s \in\{1, \ldots, A\}$ et $k \in\{1, \ldots, n\}$, on a en fait :

$$
\frac{1}{1-q^{-k}} d_{n}(1 / q)^{A-s} V_{k} \in \mathbb{Z}\left[q ; \frac{1}{q}\right]
$$

C'est ceci qui se révèle difficile, tous les termes définissant $V_{k}$ étant importants. En effet, les calculs effectués avec le logiciel Maple montrent que cela est faux si l'on remplace $V_{k}$ défini en (4.2) par $1 /(A-s) ! \times\left[d^{A-s} e_{j}(u) / d u^{A-s}\right]_{u=1}$ ou même $1 /(A-s) ! \times \sum_{j=k}^{n} q^{-k(s-1)}\left[d^{A-s} e_{j}(u) / d u^{A-s}\right]_{u=1}$.

Ecrivons maintenant notre condition suffisante nous permettant d'obtenir le Théorème 4.1 et donc le Théorème 1.1 : pour tous $s \in\{1, \ldots, A\}$ et $k \in\{1, \ldots, n\}$,

$$
\frac{1}{1-q^{-k}} \frac{d_{n}(1 / q)^{A-s}}{(A-s) !}\left[\frac{d^{A-s}}{d u^{A-s}} \sum_{j=k}^{n} e_{j}(u)\left(1-q^{n-2 j} u^{2}\right)\right]_{u=1} \in \mathbb{Z}\left[q ; \frac{1}{q}\right] .
$$

On a en effet le lemme suivant :

Lemme 4.3. Soit $A$ entier pair, et $r \in \mathbb{N}^{*}$ entier tel que $A-2 r>0$. Fixons $s \in\{1, \ldots, A\}$ et $k \in\{1, \ldots, n\}$. Si

$$
\frac{1}{1-q^{-k}} \frac{d_{n}(1 / q)^{A-s}}{(A-s) !}\left[\frac{d^{A-s}}{d u^{A-s}} \sum_{j=k}^{n} e_{j}(u)\left(1-q^{n-2 j} u^{2}\right)\right]_{u=1} \in \mathbb{Z}\left[q ; \frac{1}{q}\right]
$$

alors

$$
\frac{1}{1-q^{-k}} d_{n}(1 / q)^{A-s} V_{k} \in \mathbb{Z}\left[q ; \frac{1}{q}\right]
$$

La condition suffisante (4.6) sera démontrée par le lemme clé du paragraphe 4.3 .

Remarque 4.4. On a ainsi remplacé la somme définissant $V_{k}$ en (4.2) par $\sum_{j=k}^{n} e_{j}(u)\left(1-q^{n-2 j} u^{2}\right)$. L'intérêt de cette dernière somme est que, contrairement à celle définissant $V_{k}$, elle peut s'écrire comme limite d'une série hypergéométrique basique très bien équilibrée, qui elle-même pourra s'exprimer (via une transformation due à Andrews donnée au paragraphe 4.4) à l'aide 
d'une somme multiple $\sum_{\underline{j}} v_{j}(u)$, dont chaque terme possèdera la propriété souhaitée, à savoir :

$$
\frac{1}{1-q^{-k}} \frac{d_{n}(1 / q)^{A-s}}{(A-s) !}\left[\frac{d^{A-s}}{d u^{A-s}} v_{j}(u)\right]_{u=1} \in \mathbb{Z}\left[q ; \frac{1}{q}\right]
$$

Démonstration du Lemme 4.3. $V_{k}$ défini par (4.2) peut aussi s'écrire

$$
\begin{aligned}
V_{k}=\frac{1}{(A-s) !}\left[\frac{d^{A-s}}{d u^{A-s}} \sum_{j=k}^{n}\left(q^{-k(s-1)}-q^{-k}\right) e_{j}(u)\right]_{u=1} \\
\quad+\frac{q^{-k}}{(A-s) !}\left[\frac{d^{A-s}}{d u^{A-s}} \sum_{j=k}^{n} e_{j}(u)-(-1)^{s} e_{n-j}(u)\right]_{u=1} .
\end{aligned}
$$

Or $e_{j}(u)$ défini par (4.3) vérifie la relation

$$
e_{n-j}(u)=u^{A-4} q^{n-2 j} e_{j}(1 / u)
$$

Si l'on dérive $l$ fois cette égalité par rapport à $u$, et l'on spécialise $u=1$, alors on obtient pour des $\lambda_{i} \in \mathbb{Z}$ :

$$
\left[\frac{d^{l}}{d u^{l}} e_{n-j}(u)\right]_{u=1}=(-1)^{l} q^{n-2 j}\left[\frac{d^{l}}{d u^{l}} e_{j}(u)\right]_{u=1}+q^{n-2 j} \sum_{i=0}^{l-1} \lambda_{i}\left[\frac{d^{i}}{d u^{i}} e_{j}(u)\right]_{u=1} .
$$

De cela, nous déduisons en posant $l=A-s$ que $V_{k}$ peut s'écrire

$$
V_{k}=V_{k, 1}+V_{k, 2}+\frac{q^{-k}}{(A-s) !}\left[\frac{d^{A-s}}{d u^{A-s}} \sum_{j=k}^{n}\left(1-q^{n-2 j}\right) e_{j}(u)\right]_{u=1},
$$

avec

$$
V_{k, 1}=\left(q^{-k(s-1)}-q^{-k}\right)\left[\frac{d^{A-s}}{d u^{A-s}} \sum_{j=k}^{n} e_{j}(u)\right]_{u=1},
$$

et pour des $\left(\alpha_{i}, \beta_{i}\right) \in \mathbb{Z}^{2}$

$$
V_{k, 2}=\sum_{i=0}^{A-s-1} q^{\alpha_{i}} \beta_{i}\left[\frac{d^{i}}{d u^{i}} e_{j}(u)\right]_{u=1}
$$

Tout d'abord, concernant $V_{k, 1}$, pour tout $s \in\{1, \ldots, A\}$ on a $\left(q^{-k(s-1)}-\right.$ $\left.q^{-k}\right) /\left(1-q^{-k}\right) \in \mathbb{Z}[1 / q]$. Par ailleurs, avec le Lemme 3.3 et la relation (4.4), 
on a $: d_{n}(1 / q)^{A-s} /(A-s) ! \times\left[d^{A-s} e_{j}(u) / d u^{A-s}\right]_{u=1} \in \mathbb{Z}[q ; 1 / q]$. On en déduit que :

$$
\frac{1}{1-q^{-k}} d_{n}(1 / q)^{A-s} V_{k, 1} \in \mathbb{Z}\left[q ; \frac{1}{q}\right] \text {. }
$$

Mais on a aussi grâce au Lemme 3.3 et à la relation (4.4) $d_{n}(1 / q)^{A-s-1} V_{k, 2} \in$ $\mathbb{Z}[q ; 1 / q]$, donc à fortiori

$$
\frac{1}{1-q^{-k}} d_{n}(1 / q)^{A-s} V_{k, 2} \in \mathbb{Z}\left[q ; \frac{1}{q}\right] .
$$

Enfin on peut écrire :

$$
\begin{gathered}
\frac{1}{(A-s) !}\left[\frac{d^{A-s}}{d u^{A-s}} \sum_{j=k}^{n}\left(1-q^{n-2 j}\right) e_{j}(u)\right]_{u=1} \\
=\frac{1}{(A-s) !}\left[\frac{d^{A-s}}{d u^{A-s}} \sum_{j=k}^{n}\left(1-q^{n-2 j} u^{2}\right) e_{j}(u)\right]_{u=1}+V_{k, 3},
\end{gathered}
$$

avec $V_{k, 3}$ exactement du même type que $V_{k, 2}$, donc vérifiant

$$
\frac{1}{1-q^{-k}} d_{n}(1 / q)^{A-s} V_{k, 3} \in \mathbb{Z}\left[q ; \frac{1}{q}\right] \text {. }
$$

Finalement les expressions (4.8) et (4.11) permettent de conclure, via (4.9), (4.10) et (4.12).

\subsection{Le lemme clé}

D'après le Lemme 4.3, il suffit pour prouver le Théorème 4.1 de démontrer la condition suffisante (4.6). Celle-ci est elle-même conséquence du lemme plus général suivant :

Lemme 4.5. Soient $l, r, A, k$ et $n$ des entiers positifs, avec $A$ pair et $k \in$ $\{1, \ldots, n\}$. On $a$ :

$$
\frac{1}{1-q^{-k}} \frac{d_{n}(1 / q)^{l}}{l !}\left[\frac{d^{l}}{d u^{l}} \sum_{j=k}^{n}\left(1-q^{n-2 j} u^{2}\right) e_{j}(u)\right]_{u=1} \in \mathbb{Z}\left[q ; \frac{1}{q}\right],
$$

où $e_{j}(u)$ (défini en (4.3)) s'écrit :

$$
\begin{aligned}
e_{j}(u)= & u^{A / 2-2+(n-2 j) A / 2} q^{-r^{2} n^{2} / 2-n r / 2-j(n-j) A / 2+j} \\
& \times(-1)^{r n}\left(\frac{(q)_{r n}}{(q)_{n}^{r}}\right)^{2}\left(\frac{(q)_{n}}{\left(q u^{-1}\right)_{j}(q u)_{n-j}}\right)^{A} \frac{\left(q^{j+1} u^{-1}, q^{n+1-j} u\right)_{r n}}{(q, q)_{r n}} .
\end{aligned}
$$


Pour démontrer ce lemme, nous avons besoin d'une identité générale de transformation de séries hypergéométriques basiques, ce qui fait l'objet du paragraphe 4.4. La démonstration du Lemme 4.5 sera donnée dans le paragraphe 4.5 .

Afin d'illustrer le Lemme 4.5 lorsque $l=0$, écrivons (au signe près) $\sum_{j=k}^{n}\left(1-q^{n-2 j}\right) e_{j}(1)$, dans les cas particuliers $(A, r)=(0,0),(A, r)=(2,0)$, $(A, r)=(0,1)$ et $(A, r)=(2,1)$ respectivement (en posant $p=1 / q)$ :

$$
\begin{aligned}
& \sum_{j=k}^{n}\left(1-q^{n-2 j}\right) q^{j}=\left(1-p^{k}\right) p^{-n} \frac{1-p^{n-k+1}}{1-p} \\
& \sum_{j=k}^{n}\left(1-q^{n-2 j}\right) q^{j(j-n+1)}\left[\begin{array}{c}
n \\
j
\end{array}\right]_{q}^{2}=\left(1-p^{k}\right) p^{-k(n-k+1)}\left[\begin{array}{l}
n \\
k
\end{array}\right]_{p}\left[\begin{array}{c}
n-1 \\
k-1
\end{array}\right]_{p} \\
& \sum_{j=k}^{n}\left(1-q^{n-2 j}\right) q^{j-n(n+1) / 2}\left[\begin{array}{c}
n+j \\
n
\end{array}\right]_{q}\left[\begin{array}{c}
2 n-j \\
n
\end{array}\right]_{q} \\
& =\left(1-p^{k}\right) p^{-n(n+1) / 2}\left[\begin{array}{c}
n+k \\
n
\end{array}\right]_{p}\left[\begin{array}{c}
2 n+1-k \\
n+1
\end{array}\right]_{p} \\
& =\left(1-p^{k}\right) p^{k^{2}-k n-k-n(n-1) / 2} \sum_{l=0}^{n-k}(-1)^{l} p^{l(2 k+l-1) / 2} \\
& \times\left[\begin{array}{c}
n+k \\
k+l
\end{array}\right]_{p}\left[\begin{array}{c}
n-l-1 \\
k-1
\end{array}\right]_{p}\left[\begin{array}{c}
2 n-k-l \\
k, k
\end{array}\right]_{q}\left(\begin{array}{c}
2 n-k-k-l \\
k
\end{array}\right]_{p}
\end{aligned}
$$

L'égalité (4.13) est obtenue par un calcul direct, alors que (4.14)-(4.16) sont obtenues par des spécialisations de la transformation de Watson finie [9, Appendix III, (III.18)]. Ces identités montrent bien que $\sum_{j=k}^{n}\left(1-q^{n-2 j}\right) e_{j}(1) \in$ $\left(1-q^{-k}\right) \mathbb{Z}[q ; 1 / q]$. En particulier, (4.16) illustre le fait que ceci peut être visible malgré un membre de droite compliqué.

Lorsque l'on divise toutes ces égalités par $-(1-q)=(1-p) p^{-1}$ et l'on fait tendre $q$ vers 1 , on trouve respectivement les identités hypergéométriques 
suivantes, dont les trois premières ont été données dans [11, p. 62] :

$$
\begin{aligned}
& -\sum_{j=k}^{n}(n-2 j)=k(n-k+1), \\
& -\sum_{j=k}^{n}(n-2 j)\left(\begin{array}{c}
n \\
j
\end{array}\right)^{2}=k\left(\begin{array}{l}
n \\
k
\end{array}\right)\left(\begin{array}{l}
n-1 \\
k-1
\end{array}\right), \\
& -\sum_{j=k}^{n}(n-2 j)\left(\begin{array}{c}
n+j \\
n
\end{array}\right)\left(\begin{array}{c}
2 n-j \\
n
\end{array}\right)=k\left(\begin{array}{c}
n+k \\
k
\end{array}\right)\left(\begin{array}{c}
2 n+1-k \\
n+1
\end{array}\right), \\
& -\sum_{j=k}^{n}(n-2 j)\left(\begin{array}{c}
n+j \\
n
\end{array}\right)\left(\begin{array}{c}
2 n-j \\
n
\end{array}\right)\left(\begin{array}{c}
n \\
j
\end{array}\right)^{2} \\
& =k \sum_{l=0}^{n-k}(-1)^{l}\left(\begin{array}{c}
n+k \\
k+l
\end{array}\right)\left(\begin{array}{c}
n-l-1 \\
k-1
\end{array}\right)\left(\begin{array}{c}
2 n-k-l \\
k, n-k, n-k-l
\end{array}\right) .
\end{aligned}
$$

\subsection{Une transformation générale de $q$-séries}

Nous avons maintenant besoin, pour montrer le Lemme 4.5, d'une identité de transformation due à Andrews [1, 2] entre une somme simple très bien équilibrée et une somme multiple, qui est le $q$-analogue du Théorème 8 de [11. Cette identité a été prouvée d'abord dans [1], avant d'être vue dans [2] comme une conséquence directe du lemme de Bailey [4], qui est lui-même un outil élémentaire et très efficace pour prouver des identités de $q$-séries.

Théorème 4.6 (Andrews). Pour tous entiers $m \geq 0$ et $N \geq 0$, pour tous complexes $a, b_{1}, c_{1}, \ldots, b_{m+1}, c_{m+1}$, on $a$ :

$$
\begin{gathered}
\sum_{k=0}^{N} \frac{1-a q^{2 k}}{1-a} \frac{\left(a, b_{1}, c_{1}, \ldots, b_{m+1}, c_{m+1}, q^{-N}\right)_{k}}{\left(q, a q / b_{1}, a q / c_{1}, \ldots, a q / b_{m+1}, a q / c_{m+1}, a q^{N+1}\right)_{k}} \\
\quad \times\left(\frac{a^{m+1} q^{m+1+N}}{b_{1} c_{1} \ldots b_{m+1} c_{m+1}}\right)^{k} \\
=\frac{\left(a q, a q / b_{m+1} c_{m+1}\right)_{N}}{\left(a q / b_{m+1}, a q / c_{m+1}\right)_{N}} \sum_{0 \leq l_{1} \leq \cdots \leq l_{m} \leq N} \frac{a^{l_{1}+\cdots+l_{m-1}} q^{l_{1}+\cdots+l_{m}}}{\left(b_{2} c_{2}\right)^{l_{1}} \ldots\left(b_{m} c_{m}\right)^{l_{m-1}}} \\
\quad \times \frac{\left(q^{-N}\right)_{l_{m}}}{\left(b_{m+1} c_{m+1} q^{-N} / a\right)_{l_{m}}} \prod_{i=1}^{m} \frac{\left(b_{i+1}, c_{i+1}\right)_{l_{i}}}{\left(a q / b_{i}, a q / c_{i}\right)_{l_{i}}} \frac{\left(a q / b_{i} c_{i}\right)_{l_{i}-l_{i-1}}}{(q)_{l_{i}-l_{i-1}}}
\end{gathered}
$$

Cette égalité justifie notre intention aux paragraphes précédants de nous ramener à une série très bien équilibrée. En effet, lorsque pour tout $i \in$ 
$\{1, \ldots, m+1\}, a q / b_{i} \neq q^{-N}$ et $a q / c_{i} \neq q^{-N}$, le membre de gauche de (4.17) peut s'écrire en terme de série hypergéométrique basique très bien équilibrée :

$$
2 m+6 \phi_{2 m+5}\left[\begin{array}{c}
a, q \sqrt{a},-q \sqrt{a}, b_{1}, c_{1}, \ldots, b_{m+1}, c_{m+1}, q^{-N} \\
\sqrt{a},-\sqrt{a}, a q / b_{1}, a q / c_{1}, \ldots, a q / b_{m+1}, a q / c_{m+1}, a q^{N+1} ; q, z
\end{array}\right],
$$

avec $z=a^{m+1} q^{m+1+N} / b_{1} c_{1} \ldots b_{m+1} c_{m+1}$. Cependant, si l'un des facteurs $b_{i}$ ou $c_{i}$, par exemple $b_{1}$, s'écrit $a q^{1+N}$, alors la série hypergéométrique cidessus n'est plus une somme finie. En effet, le facteur $\left(q^{-N}\right)_{k}$ du numérateur, garantissant cette finitude, se simplifie avec le dénominateur $\left(a q / b_{1}\right)_{k}$. Le membre de gauche de (4.17), qui est toujours une somme finie, peut alors être vu comme limite d'une série hypergéométrique basique très bien équilibrée :

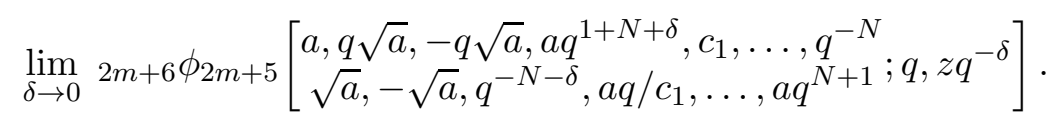

Remarque 4.7. Dans le cas $m=1$, l'égalité (4.17) est exactement la transformation finie de Watson déjà mentionnée dans le paragraphe précédent et utile pour prouver les identités (4.14)-(4.16). Ceci indique bien que (4.17) pourrait être l'ingrédient manquant pour démontrer le Lemme 4.5.

\subsection{Démonstration du Lemme 4.5}

Posons $v_{j}(u):=e_{j}(u)\left(1-q^{n-2 j} u^{2}\right)$, de sorte que le crochet de l'expression que l'on étudie dans le Lemme 4.5 peut s'écrire

$$
\sum_{j=k}^{n}\left(1-q^{n-2 j} u^{2}\right) e_{j}(u)=\sum_{j=k}^{n} v_{j}(u)=\sum_{j=0}^{n-k} v_{k+j}(u) .
$$

Ceci se transforme en

$$
\begin{aligned}
v_{k}(u) \times \sum_{j=0}^{n-k} p^{j((A / 2-r) n+A / 2-1)} \times \frac{1-a p^{2 j}}{1-a} & \\
& \times \frac{\left(a, p, p^{r n+k+1} u, p^{k-n} u, \ldots, p^{k-n} u, p^{k+1} u^{2}, p^{k-n} ; p\right)_{j}}{\left(p, a, p^{k-r n-n} u, p^{k+1} u, \ldots, p^{k+1} u, p^{k-n}, p^{k+1} u^{2} ; p\right)_{j}},
\end{aligned}
$$

avec $p=1 / q$ et $a=p^{2 k-n} u^{2}$, les facteurs $p^{k-n} u$ au numérateur et $p^{k+1} u$ au dénominateur apparaissant $A+1$ fois. On peut maintenant appliquer la formule d'Andrews (4.17) à (4.18) en remplaçant $q$ par $p$, et en posant $N=n-k, m=A / 2+1, a=p^{2 k-n} u^{2}, b_{1}=p^{r n+k+1} u, c_{1}=b_{2}=c_{2}=\cdots=$ 
$b_{A / 2}=c_{A / 2}=b_{A / 2+1}=c_{A / 2+2}=p^{k-n} u, c_{A / 2+1}=p^{k+1} u^{2}$ et $b_{A / 2+2}=p$. Ceci donne comme expression alternative pour (4.18) :

$$
\begin{aligned}
v_{k}(u) \times & \frac{\left(p^{2 k+1-n} u^{2}, p^{k} u ; p\right)_{n-k}}{\left(p^{2 k-n} u^{2}, p^{k+1} u ; p\right)_{n-k}} \\
& \times \sum_{0 \leq l_{1} \leq \cdots \leq l_{A / 2+1} \leq n-k} \frac{\left(p^{2 k-n} u^{2}\right)^{l_{1}+\cdots+l_{A / 2}} p^{l_{1}+\cdots+l_{A / 2+1}}}{\left(p^{2 k-2 n} u^{2}\right)^{l_{1}+\cdots+l_{A / 2}-1}\left(p^{2 k-n+1} u^{3}\right)^{l_{A / 2}}} \\
& \times \frac{\left(p^{-r n}, p^{k-n} u, p^{k-n} u ; p\right)_{l_{1}}}{\left(p, p^{k-n-r n} u, p^{k+1} u ; p\right)_{l_{1}}} \times \prod_{i=1}^{A / 2} \frac{\left(p^{k-n} u, p^{k-n} u ; p\right)_{l_{i}}\left(p^{n+1} ; p\right)_{l_{i}-l_{i-1}}}{\left(p^{k+1} u, p^{k+1} u ; p\right)_{l_{i}}(p ; p)_{l_{i}-l_{i-1}}} \\
\times & \frac{\left(p^{k+1} u^{2} ; p\right)_{l_{A / 2}}}{\left(p^{k-n} u ; p\right)_{l_{A / 2}}} \times \frac{\left(p^{k-n}, p, p^{k-n} u ; p\right)_{l_{A / 2+1}}}{\left(p^{1-n} / u, p^{k+1} u, p^{k-n} ; p\right)_{l_{A / 2+1}}} \times \frac{(1 / u ; p)_{l_{A / 2+1}-l_{A / 2}}}{(p ; p)_{l_{A / 2+1}-l_{A / 2}}} .
\end{aligned}
$$

En posant $\underline{l}:=\left(l_{1}, \ldots, l_{A / 2+1}\right)$, on obtient après maintes transformations élémentaires de $p$-factoriels $(p=1 / q)$ :

$$
\sum_{j=k}^{n}\left(1-q^{n-2 j} u^{2}\right) e_{j}(u)=\left(1-p^{k} u\right) \sum_{0 \leq l_{1} \leq \cdots \leq l_{A / 2+1} \leq n-k} w_{\underline{l}}(u),
$$

où pour $\xi(\underline{l}), \varphi(\underline{l})$ et $\psi(\underline{l})$ éléments de $\mathbb{Z}$,

$$
\begin{aligned}
& w_{\underline{l}}(u):=(-1)^{\xi(\underline{l})} p^{\varphi(\underline{l})} u^{\psi(\underline{l})} \times R_{1}(n, k, n, p, u) \times \prod_{i=2}^{A / 2}\left[\begin{array}{c}
n+l_{i}-l_{i-1} \\
k+l_{i}
\end{array}\right]_{p} \\
& \times\left[\begin{array}{c}
l_{A / 2+1} \\
l_{1}, l_{2}-l_{1}, \ldots, l_{A / 2+1}-l_{A / 2}
\end{array}\right]_{p} \times \prod_{i=1}^{A / 2} R_{0}\left(0, n+1, k+l_{i}, p, 1 / u\right) \\
& \times \prod_{i=2}^{A / 2} R_{0}\left(0, n-k-l_{i-1}+1,0, p, 1 / u\right) \times R_{0}\left(0, k+l_{i}+1,0, p, u\right) \\
& \times R_{1}\left(n, n-l_{1}-k, n-l_{1}, p, 1 / u\right) \times R_{2}\left(n, k, l_{A / 2+1}, l_{A / 2}, p, u\right),
\end{aligned}
$$

et pour $\alpha \leq \gamma<\beta$ entiers et $|q| \neq 1$

$$
R_{0}(\alpha, \beta, \gamma, q, u):=\frac{(1-u)(q)_{\beta-\alpha-1}}{\left(q^{\alpha-\gamma} u\right)_{\beta-\alpha}}=\frac{(q)_{\beta-\alpha-1}}{\left(q^{\alpha-\gamma} u\right)_{\gamma-\alpha}(q u)_{\beta-\gamma-1}},
$$

puis pour $n \geq j \geq i \geq 0$ entiers et $|q| \neq 1$

$$
R_{1}(n, i, j, q, u):=\frac{(q)_{r n}}{(q)_{n}^{r}} \times \frac{\left(q^{n+i-j+1} u\right)_{r n-n+j}}{(q)_{r n-n+j}},
$$


et enfin pour $0 \leq m_{2} \leq m_{1} \leq n-k$ entiers et $|q| \neq 1$

$$
R_{2}\left(n, k, m_{1}, m_{2}, q, u\right):=\frac{(q)_{n}}{(q u)_{n}} \frac{(1 / u)_{m_{1}-m_{2}}}{\left(q u^{2}\right)_{k-1}} \frac{\left(q u^{2}\right)_{k+m_{2}}}{(q u)_{k+m_{1}}} \frac{(q u)_{n-m_{1}-1}}{(q / u)_{n-k-m_{1}}} .
$$

Remarque 4.8. Les fractions $R_{0} /(1-u), R_{1}$ et $R_{2}$ définies à l'aide de (4.21)(4.23) sont des $q$-analogues de certaines briques élémentaires et spéciales de 11].

L'expression (4.19) indique que pour démontrer le Lemme 4.5, il nous suffit maintenant, en vertu de la formule de Leibniz de dérivation d'un produit, de prouver que pour tout entier $l$ :

$$
\frac{d_{n}(p)^{l}}{l !}\left[\frac{d^{l}}{d u^{l}} w_{\underline{l}}(u)\right]_{u=1} \in \mathbb{Z}\left[p ; \frac{1}{p}\right]=\mathbb{Z}\left[q ; \frac{1}{q}\right] .
$$

Mais grâce à la formule de Leibniz de nouveau et à l'expression (4.20), ceci est une conséquence du lemme suivant :

Lemme 4.9. Soit $|q| \neq 1$. Pour tous entiers $l \geq 0, \alpha \leq \gamma<\beta, n \geq j \geq i \geq 0$ et $0 \leq m_{2} \leq m_{1} \leq n-k$, on $a$ :

$$
\begin{aligned}
& \frac{d_{\beta-\alpha-1}(q)^{l}}{l !}\left[\frac{d^{l}}{d u^{l}} R_{0}(\alpha, \beta, \gamma, q, u)\right]_{u=1} \in \mathbb{Z}\left[q, \frac{1}{q}\right], \\
& \frac{d_{n}(q)^{l}}{l !}\left[\frac{d^{l}}{d u^{l}} R_{1}(n, i, j, q, u)\right]_{u=1} \in \mathbb{Z}\left[q, \frac{1}{q}\right], \\
& \frac{d_{n}(q)^{l}}{l !}\left[\frac{d^{l}}{d u^{l}} R_{2}\left(n, k, m_{1}, m_{2}, q, u\right)\right]_{u=1} \in \mathbb{Z}\left[q, \frac{1}{q}\right] .
\end{aligned}
$$

De plus, ces propriétés restent valables lorsque u est remplacé par $u^{e}, e \in \mathbb{Z}$ (en particulier par $1 / u$ ).

Démonstration. Tout d'abord, le fait que les propriétés (4.24)-(4.26) restent valables lorsque $u$ est remplacé par $u^{e}, e \in \mathbb{Z}$, est une simple conséquence de la dérivation des fonctions composées.

Commençons donc par démontrer (4.24), en décomposant en éléments simples la fraction rationnelle (en $u$ ) :

$$
R_{0}(\alpha, \beta, \gamma, q, u)=\sum_{\substack{j=\alpha \\ j \neq \gamma}}^{\beta-1} a_{j} \frac{1-q^{j-\gamma}}{1-q^{j-\gamma} u}
$$


avec

$$
a_{j}=\frac{(1-q) \ldots\left(1-q^{\beta-\alpha-1}\right)}{\left(1-q^{\alpha-j}\right) \ldots\left(1-q^{-1}\right)(1-q) \ldots\left(1-q^{\beta-j-1}\right)},
$$

qui se récrit

$$
a_{j}=(-1)^{j-\alpha} q^{(j-\alpha)(j-\alpha+1) / 2}\left[\begin{array}{c}
\beta-\alpha-1 \\
j-\alpha
\end{array}\right]_{q} \in \mathbb{Z}[q]
$$

En dérivant, on obtient donc

$$
\frac{1}{l !} \frac{d^{l}}{d u^{l}} R_{0}(\alpha, \beta, \gamma, q, u)=\sum_{\substack{j=\alpha \\ j \neq \gamma}}^{\beta-1} q^{(j-\gamma) l} a_{j} \frac{1-q^{j-\gamma}}{\left(1-q^{j-\gamma} u\right)^{l+1}}
$$

ce qui en prenant $u=1$ prouve (4.24).

Démontrons ensuite (4.25). Comme $R_{1}(n, i, j, q, u)$ est un polynôme en $u$ de degré $(r-1) n+j$, on a $\frac{d^{l}}{d u^{l}} R_{1}(n, i, j, q, u)=0$ si $l>(r-1) n+j$. Supposons donc que $l \leq(r-1) n+j$. On a alors :

$$
\begin{aligned}
\frac{1}{l !} \frac{d^{l}}{d u^{l}} R_{1}(n, i, j, q, u)=\sum_{n+i-j+1 \leq f_{1}<\cdots<f_{l} \leq r n+i} & (-1)^{l} q^{f_{1}+\cdots+f_{l}} \\
& \times \frac{R_{1}(n, i, j, q, u)}{\left(1-q^{f_{1}} u\right) \ldots\left(1-q^{f_{l}} u\right)} .
\end{aligned}
$$

En posant $g_{m}:=f_{m}-(n+i-j)$ pour tout $m \in\{1, \ldots, l\}$, ceci peut s'écrire lorsque $u=1$ :

$$
\frac{1}{l !}\left[\frac{d^{l}}{d u^{l}} R_{1}(n, i, j, q, u)\right]_{u=1}=\sum_{1 \leq g_{1}<\cdots<g_{l} \leq r n-n+j}(-1)^{l} q^{(n+i-j) l+g_{1}+\cdots+g_{l}} \tilde{R}_{1}(q),
$$

avec

$$
\tilde{R}_{1}(q):=\frac{(q)_{r n}}{(q)_{n}^{r}} \frac{\left(q^{n+i-j+1}\right)_{r n-n+j}}{(q)_{r n-n+j}} \frac{1}{\left(1-q^{n+i-j+g_{1}}\right) \ldots\left(1-q^{n+i-j+g_{l}}\right)} .
$$

Pour prouver (4.25), il suffit donc de démontrer que

$$
d_{n}(q)^{l} \tilde{R}_{1}(q) \in \mathbb{Z}[q]
$$

Pour cela, faisons quelques rappels sur les polynômes cyclotomiques. Pour $t \in \mathbb{N}$, le $t$-ième polynôme cyclotomique est $\phi_{t}(x):=\prod_{k \wedge t=1, k \leq t}\left(x-\mathrm{e}^{2 i k \pi / t}\right)$, 
avec comme première propriété le fait que $\phi_{t}(x) \in \mathbb{Z}[x]$. Ensuite, on peut montrer que

$$
q^{n}-1=\prod_{d \mid n} \phi_{d}(q)
$$

ce qui permet de déduire d'une part que

$$
d_{n}(q)=\prod_{t=1}^{n} \phi_{t}(q)
$$

et d'autre part l'ordre de divisibilité suivant dans $\mathbb{Z}[q]$ :

$$
\operatorname{ord}_{\phi_{t}(q)}\left(\prod_{l=1}^{n} \frac{q^{l}-1}{q-1}\right)=\left\{\begin{array}{l}
0 \text { si } t=1 \\
\left\lfloor\frac{n}{t}\right\rfloor \text { si } t \geq 2 .
\end{array}\right.
$$

Maintenant, comme $\tilde{R}_{1}(q)$ est une fraction rationnelle en $q$, dont numérateur et dénominateur s'expriment au signe près en produits de fonctions du type $\phi_{t}(q)$ (de par (4.29) ), il suffit pour prouver (4.28) de voir que

$$
\forall t \in \mathbb{N}, \operatorname{ord}_{\phi_{t}(q)}\left(d_{n}(q)^{l} \tilde{R}_{1}(q)\right) \geq 0 .
$$

Nous distinguons pour cela trois cas.

- Si $t=1$, alors on a en vertu de (4.30) et (4.31)

$$
\operatorname{ord}_{\phi_{1}(q)}\left(d_{n}(q)^{l} \tilde{R}_{1}(q)\right)=l+\operatorname{ord}_{\phi_{1}(q)}\left(\tilde{R}_{1}(q)\right)=l-l=0 .
$$

- Si $2 \leq t \leq n$, alors (4.30) et (4.31) donnent

$$
\begin{aligned}
& \operatorname{ord}_{\phi_{t}(q)}\left(d_{n}(q)^{l} \tilde{R}_{1}(q)\right)=\sum_{m=1}^{l}\left(1-\operatorname{ord}_{\phi_{t}(q)}\left(1-q^{g_{m}+n+i-j}\right)\right) \\
& +\left(\left\lfloor\frac{r n}{t}\right\rfloor-r\left\lfloor\frac{n}{t}\right\rfloor\right)+\left(\left\lfloor\frac{r n+i}{t}\right\rfloor-\left\lfloor\frac{n+i-j}{t}\right\rfloor-\left\lfloor\frac{r n-n+j}{t}\right\rfloor\right),
\end{aligned}
$$

où il est facile de voir que le terme à l'intérieur de chaque parenthèse est positif ou nul, ce qui permet de conclure.

- Si $t>n$, on a dans un premier temps par (4.30)

$$
\operatorname{ord}_{\phi_{t}(q)}\left(d_{n}(q)^{l} \tilde{R}_{1}(q)\right)=\operatorname{ord}_{\phi_{t}(q)}\left(\tilde{R}_{1}(q)\right) .
$$


D'autre part, puisque $1 \leq g_{1}<\cdots<g_{l} \leq r n-n+j$, on peut écrire en utilisant (4.29)

$$
\begin{gathered}
\sum_{m=1}^{l} \operatorname{ord}_{\phi_{t}(q)}\left(1-q^{g_{m}+n+i-j}\right) \leq \sum_{\substack{g=n+i-j+1 \\
g=n+i-j+1 \\
t \mid g}}^{r n+i} \operatorname{ord}_{\phi_{t}(q)}\left(1-q^{g}\right)=\sum_{\substack{g=n+i-j+1 \\
t \mid g}}^{r n+i} 1=\left\lfloor\frac{r n+i}{t}\right\rfloor-\left\lfloor\frac{n+i-j}{t}\right\rfloor \\
=\operatorname{ord}_{\phi_{t}(q)}\left(\left(q^{n+i-j+1}\right)_{r n-n+j}\right) .
\end{gathered}
$$

Donc finalement, puisque $n \geq j$ et $t>n$, on a en reprenant (4.27)

$$
\begin{array}{r}
\operatorname{ord}_{\phi_{t}(q)}\left(d_{n}(q)^{l} \tilde{R}_{1}(q)\right) \geq\left\lfloor\frac{r n}{t}\right\rfloor-r\left\lfloor\frac{n}{t}\right\rfloor-\left\lfloor\frac{r n-n+j}{t}\right\rfloor \\
=\left\lfloor\frac{r n}{t}\right\rfloor-\left\lfloor\frac{r n-n+j}{t}\right\rfloor \geq 0 .
\end{array}
$$

Il ne reste plus qu'à prouver (4.26). Supposons dans un premier temps que $m_{1}>m_{2}$. On peut alors écrire en reprenant la définition (4.23)

$$
R_{2}\left(n, k, m_{1}, m_{2}, q, u\right)=\left(1-u^{-1}\right) R_{3}(q, u)
$$

où

$$
R_{3}(q, u):=\frac{(q)_{n}}{(q u)_{n}} \frac{(q / u)_{m_{1}-m_{2}-1}}{\left(q u^{2}\right)_{k-1}} \frac{\left(q u^{2}\right)_{k+m_{2}}}{(q u)_{k+m_{1}}} \frac{(q u)_{n-m_{1}-1}}{(q / u)_{n-k-m_{1}}} .
$$

Ceci montre, à l'aide de la dérivation des produits par Leibniz, qu'il suffit pour prouver (4.26) de montrer que

$$
\frac{d_{n}(q)^{l+1}}{l !}\left[\frac{d^{l}}{d u^{l}} R_{3}(q, u)\right]_{u=1} \in \mathbb{Z}\left[q, \frac{1}{q}\right] .
$$

Mais, sachant que $m_{1}-m_{2}-1 \leq n$ et $k+m_{2} \leq k+m_{1} \leq n$, la propriété de Leibniz et la dérivation des fonctions composées permettent de voir qu'il suffit pour montrer (4.33) de prouver que pour tous $1 \leq h_{1} \leq \cdots \leq h_{l} \leq n$

$$
R_{4}(q):=d_{n}(q)^{l+1} \frac{R_{3}(q, 1)}{\left(1-q^{h_{1}}\right) \ldots\left(1-q^{h_{l}}\right)} \in \mathbb{Z}[q]
$$

On distingue les trois mêmes cas que précédemment. Si $t=1$ ou $t>n$, on voit par (4.30) et (4.31) que $\operatorname{ord}_{\phi_{t}(q)}\left(R_{4}(q)\right)=0$. Dans le cas où $2 \leq t \leq n$, 
on écrit

$$
\begin{aligned}
& \operatorname{ord}_{\phi_{t}(q)}\left(R_{4}(q)\right)=1+\sum_{m=1}^{l}\left(1-\operatorname{ord}_{\phi_{t}(q)}\left(1-q^{h_{m}}\right)\right)+\operatorname{ord}_{\phi_{t}(q)}\left(R_{3}(q, 1)\right) \\
& \geq 1+\operatorname{ord}_{\phi_{t}(q)}\left(R_{3}(q, 1)\right)=1+\left\lfloor\frac{m_{1}-m_{2}-1}{t}\right\rfloor-\left\lfloor\frac{k-1}{t}\right\rfloor \\
&+\left\lfloor\frac{k+m_{2}}{t}\right\rfloor-\left\lfloor\frac{k+m_{1}}{t}\right\rfloor+\left\lfloor\frac{n-m_{1}-1}{t}\right\rfloor-\left\lfloor\frac{n-k-m_{1}}{t}\right\rfloor \\
&= 1+\left(\left\lfloor\frac{n-m_{1}-1}{t}\right\rfloor-\left\lfloor\frac{n-k-m_{1}}{t}\right\rfloor-\left\lfloor\frac{k-1}{t}\right\rfloor\right) \\
&-\left(\left\lfloor\frac{k+m_{1}}{t}\right\rfloor-\left\lfloor\frac{k+m_{2}}{t}\right\rfloor-\left\lfloor\frac{m_{1}-m_{2}}{t}\right\rfloor\right) \\
&-\left(\left\lfloor\frac{m_{1}-m_{2}}{t}\right\rfloor-\left\lfloor\frac{m_{1}-m_{2}-1}{t}\right\rfloor\right) .
\end{aligned}
$$

Or l'identité élémentaire $0 \leq\lfloor a+b\rfloor-\lfloor a\rfloor-\lfloor b\rfloor \leq 1$ permet d'affirmer que la première parenthèse de cette expression est supérieure ou égale à 0 , et les deux suivantes sont entre 0 et 1 . Ceci montre que si $\operatorname{ord}_{\phi_{t}(q)}\left(R_{4}(q)\right)<0$, alors les deux dernières parenthèses doivent valoir 1 , ce qui implique que $t$ divise $m_{1}-m_{2}$. Mais en écrivant ces deux dernières parenthèses de deux autres façons, on voit que $\operatorname{ord}_{\phi_{t}(q)}\left(R_{4}(q)\right)<0$ impliquerait aussi le fait que $t$ divise $k+m_{2}+1$ et $k+m_{1}$; donc $t$ diviserait $1=\left(m_{1}-m_{2}\right)+\left(k+m_{2}+1\right)-\left(k+m_{1}\right)$, ce qui est absurde car $t \geq 2$. Donc $\operatorname{ord}_{\phi_{t}(q)}\left(R_{4}(q)\right) \geq 0$.

Démontrons enfin (4.26) dans le cas $m_{1}=m_{2}$. De même que ci-dessus, il suffit de montrer que pour tous $1 \leq h_{1} \leq \cdots \leq h_{l} \leq n$

$$
R_{5}(q):=d_{n}(q)^{l} \frac{R_{2}\left(n, k, m_{1}, m_{1}, q, 1\right)}{\left(1-q^{h_{1}}\right) \ldots\left(1-q^{h_{l}}\right)} \in \mathbb{Z}[q] .
$$

Ici aussi si $t=1$ ou $t>n$, alors par (4.30) et (4.31), $\operatorname{ord}_{\phi_{t}(q)}\left(R_{5}(q)\right)=0$. Pour $2 \leq t \leq n$, l'ordre de multiplicité $\operatorname{ord}_{\phi_{t}(q)}\left(R_{5}(q)\right)$ vaut

$$
\begin{aligned}
\sum_{m=1}^{l}(1 & \left.-\operatorname{ord}_{\phi_{t}(q)}\left(1-q^{h_{m}}\right)\right)+\operatorname{ord}_{\phi_{t}(q)}\left(R_{2}\left(n, k, m_{1}, m_{1}, q, 1\right)\right) \\
& \geq \operatorname{ord}_{\phi_{t}(q)}\left(R_{2}\left(n, k, m_{1}, m_{1}, q, 1\right)\right) \\
& =\left\lfloor\frac{n-m_{1}-1}{t}\right\rfloor-\left\lfloor\frac{k-1}{t}\right\rfloor-\left\lfloor\frac{n-k-m_{1}}{t}\right\rfloor \geq 0 .
\end{aligned}
$$




\section{Bibliographie}

[1] Andrews (G.E.), Problems and prospects for basic hypergeometric functions, Theory and application for basic hypergeometric functions, R. A. Askey, ed., Math. Res. Center, Univ. Wisconsin, Publ. No. 35, Academic Press, New York (1975), 191-224.

[2] Andrews (G.E.), Multiple series Rogers-Ramanujan type identities, Pacific J. Math., Vol. 114, no. 2 (1984), 267-283.

[3] Apéry (R.), Irrationalité de $\zeta(2)$ et $\zeta(3)$, Astérisque 61 (1979), 11-13.

[4] W. N. Bailey, Identities of the Rogers-Ramanujan type, Proc. London Math. Soc. (2) 50 (1949), 1-10.

[5] Borwein (P.), On the irrationality of certain series, Math. Proc. Cambridge Philos. Soc. 112.1 (1992), 141-146.

[6] Ball (K.) et Rivoal (T.), Irrationalité d'une infinité de valeurs de la fonction zêta aux entiers impairs, Invent. Math. 146. 1 (2001), 193-207.

[7] Bundschun (P.) et VäÄnÄnen (K.), Arithmetical investigations of a certain infinite product, Compositio Math. 91.2 (1994), 175-199.

[8] Fischler (S.), Irrationalité de valeurs de zêta (d'après Apéry, Rivoal, ...), Sem. Bourbaki 2002-2003, exposé no. 910, Astérisque 294 (2004), 27-62.

[9] Gasper (G.) and Rahman (M.), Basic Hypergeometric Series, 2nd Edition, Encyclopedia of mathematics and its applications, Vol. 96, Cambridge University Press, Cambridge, 2004.

[10] Kaneko (M.), Kurokawa (N.) et Wakayama (M.), A variation of Euler's approach to values of the Riemann zeta function, Kyushu J. Math., Vol. 57.1 (2003), 175-192.

[11] Krattenthaler (C.) et Rivoal (T.), Hypergéométrie et Fonction Zêta de Riemann, Mem. Amer. Math. Soc., Vol. 186, no. 875 (2007), 1-87.

[12] Krattenthaler (C.), Rivoal (T.) et Zudilin (W.), Séries hypergéométriques basiques, q-analogues des valeurs de la fonction zêta et séries d'Eisenstein, J. Inst. Jussieu 5.1 (2006), 53-79.

[13] Nesterenko (Yu. V.), On the linear independance of numbers, (en russe) Vest. Mosk. Univ., Ser. I, no. 1 (1985), 46-54; trad. en anglais dans Mosc. Univ. Math. Bull. 40.1 (1985), 69-74.

[14] Nesterenko (Yu. V.), Modular functions and transcendance questions, (en russe) Math. Sb. 187.9 (1996), 65-96; trad. en anglais dans Sb. Math. 187.9 (1996), 1319-1348.

[15] Postelmans (K.) et Van Assche (W.), Irrationality of $\zeta_{q}(1)$ and $\zeta_{q}(2)$, J. Number Theory 126 (2007), 119-154.

[16] Rivoal (T.), La fonction zêta de Riemann prend une infinité de valeurs irrationnelles aux entiers impairs, C. R. Acad. Sci. Paris, Série I Math. 331.4 (2000), 267-270. 
[17] Rivonl (T.), Propriétés diophantiennes des valeurs de la fonction zêta de Riemann aux entiers impairs, thèse de doctorat, Université de Caen, 2001. http://theses-EN-ligne.in2p3.fr.

[18] Rivoal (T.), Irrationalité d'au moins un des neuf nombres $\zeta(5), \zeta(7), \ldots, \zeta(21)$, Acta Arith. 103.2 (2002), 157-167.

[19] Serre (J. P.), Cours d'arithmétique, Presses Universitaires de France, Paris, 1970.

[20] Stanley (R. P.), Enumerative Combinatorics, Vol. 1, Cambridge University Press, Cambridge, 1998.

[21] VAn Assche (W.), Little q-Legendre polynomials and irrationality of certain Lambert series, Ramanujan J. 5.3 (2001), 295-310.

[22] Zudilin (W.), Arithmetic of linear forms involving odd zeta values, J. Théor. Nombres Bordeaux 16.1 (2004), 251-291.

[23] Zudilin (W.), Diophantine problems for q-zeta values, (en russe) Mat. Zametki, 72.6 (2002), 936-940; trad. en anglais dans Math. Notes 72.6 (2002), 858-862.

Frédéric Jouhet, Université de Lyon, Université Lyon I, Institut Camille Jordan, UMR 5208, 43, bd du 11 Novembre 1918, 69622 Villeurbanne Cedex, France jouhet@math.univ-lyon1.fr

Elie Mosaki,

Université de Lyon, Université Lyon I, Institut Camille Jordan, UMR 5208, 43, bd du 11 Novembre 1918, 69622 Villeurbanne Cedex, France

mosaki@math.univ-lyon1.fr 